\title{
Femtosecond wave packet spectroscopy: Coherences, the potential, and structural determination
}

\author{
M. Gruebele $e^{\text {a) }}$ and A. H. Zewail \\ Arthur Amos Noyes Laboratory of Chemical Physics, California Institute of Technology, Pasadena, \\ California 91125
}

(Received 7 July 1992; accepted 5 October 1992)

\begin{abstract}
Recently, we presented a formalism for extracting highly resolved spectral information and the potential of bound isolated systems from coherent ultrafast laser experiments, using $I_{2}$ as a model system [Gruebele et al., Chem. Phys. Lett. 166, 459 (1990)]. The key to this approach is the formation of coherent wave packets on the potential energy curve (or surface) of intercst, and the measurement of their scalar and vector properties. Here we give a full account of the method by analyzing the coherences of the wave packet in the temporal transients of molecules excited by ultrashort laser pulses, either at room temperature, or in a molecular beam. From this, some general considerations for properly treating temporal data can be derived. We also present a direct inversion to the potential and quantum and classical calculations for comparison with the experiments.
\end{abstract}

\section{INTRODUCTION}

The creation of coherent wave packets by an ultrashort laser pulse is essential for time-resolved studies of the nuclear motion in chemical reactions. ${ }^{1}$ For nonreactive isolated systems, these packets are in bound (or quasibound) potentials, where they may undergo periodic oscillations, dephasing, and recurrence. A question of interest, particularly for structural determination, is the following: How can such an ultrashort pulse "see" the vibrational/ rotational eigenstates of a bound potential? The key is that the pulse has sufficient bandwidth to form a coherent superposition of eigenstates with a well-defined phase evolution. The resulting wave packet shows some spatial localization, and if its motion can be observed temporally, all the spectroscopic information (e.g., vibrational and rotational level structure) can be obtained. With picosecond pulses, coherence has been probed in isolated molecules to study rotational dynamics (structural determination) and intramolecular vibrational energy redistribution (IVR). ${ }^{2}$ With femtosecond pulses, a much broader range of vibrational and rotational dynamics becomes accessible, due to the formation of larger bandwidth wave packets. ${ }^{3}$

The time resolution is now sufficient to separate the nuclear vibrational and rotational motions. Just as NMR, ESR, microwave, and visible spectroscopy have benefited from pulsed techniques (in the form of FT-NMR, FTESR, FT-microwave, and quantum beat ${ }^{4}$ spectroscopies) with bandwidths in the $\mathrm{kHz}$ to $\mathrm{GHz}$ region, it is now possible to open a spectral window in the $\mathrm{THz}$ region with lasers ranging from the ir to the near uv and beyond.

Previously, we addressed the question of how highly resolved spectroscopic information can be obtained from fs pulse excitation despite their broad spectral bandwidth, by studying the coherences observed in the transient. ${ }^{5,6}$ In the following, we will consider the model system $I_{2}$ (in the $B$

\footnotetext{
2) Present address: School of Chemical Sciences, University of Illinois at Urbana-Champaign, 505 So. Mathews Ave., Urbana, IL 61801.
}

electronic state) and compare theory with experiments. The high-resolution spectroscopy of $I_{2}$ is well known, ${ }^{7}$ and rovibrational wave packets can easily be created; ${ }^{8}$ this molecule is therefore an ideal benchmark for the type of information that can be extracted by time-resolved techniques. We show that vibrational and rotational coherences can be separated experimentally. The wave packet dynamics yield direct information about the vibrational and rotational motion (as well as rovibrational couplings), allowing one to obtain the potential. With a small number of such femtosecond measurements (less than ten in the present case) spanning a wide range of the spectrum (from the term energy to the dissociation limit of the $B$ state in the present case), we are able to obtain excellent agreement with results in the literature. As with rotational coherence spectroscopy, the wave packet approach promises to be powerful for bound potentials, especially in larger molecular systems.

The results illustrate two points. First, the spectroscopy and potentials of bound systems can be obtained from wave packet dynamics with a separation of time scales for the vibrational and rotational motions. This separation, which can also be made by measurements of the scalar and vector properties of the dynamics, simplifies the analysis considerably. Also, coherences are formed only on the potential of interest and there are no complications from the other states involved in the transitions. Second, the correspondence made here between high resolution spectra and time-resolved spectra is valid if one is considering simple systems. For chemical reactions with intermediates or large complex systems, this correspondence is not as valid in practice.

We will first outline the experimental approach, and then provide a general description of the wave packet motion for the case at hand. To apply them to real systems, several simplifications can first be made which greatly enhance the usefulness of the formalism and the physical picture to be gleaned from it. With these considerations, we are then equipped to consider applications to real systems. 


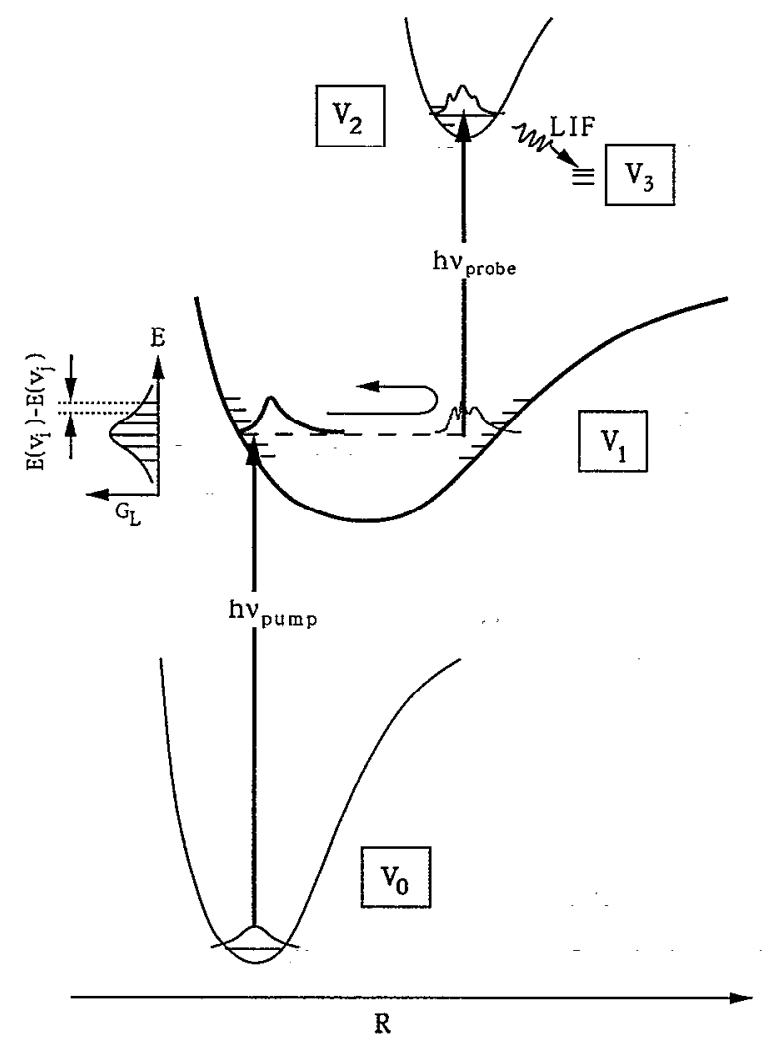

FIG. 1. Schematic of the femtosecond pump-probe experiment for a bound potential; the wave packet formed on the intermediate surface $\left(V_{1}\right)$ consists of a number of eigenstates covered by the laser bandwidth $G_{L}$, and with characteristic frequencies $E\left(v_{i}\right)-E\left(v_{j}\right)$; these frequencies are preserved in the coherence of the transient obtained by probing the packet to a final surface $\left(V_{2}\right)$, from where it can be detected by LIF to $V_{3}$ (or through mass spectrometry or other means).

We conclude with time-dependent quantum calculations, to show how molecular rotation and vibration under beam or gas-cell conditions affect the observed coherence.

\section{THE FEMTOSECOND WAVE PACKET APPROACH}

The methodology of the experiments has been described in detail elsewhere. ${ }^{8}$ Here we discuss the physical picture of the laser-molecule interactions, and the appearance of the time-resolved transients obtained.

Figure 1 shows the potential energy function for $\mathrm{I}_{2}$, for a typical pump-probe experiment with an initial pulse at $550 \mathrm{~nm}$ and a second pulse at $310 \mathrm{~nm}$. The experiment starts from an incoherent superposition of initial states, described in the simplest case by a single temperature, and requiring in general a number of "effective" translational/ rotational/vibrational temperatures or a more general density matrix description. This incoherent superposition is pumped by an ultrashort pulse of bandwidth $\Delta \omega$ (coherence width of a transform-limited pulse) into a coherent superposition, resulting in a wave packet (or more properly, a partially coherent "density-matrix packet") moving on the excited state surface (the $B$ state, in $\mathrm{I}_{2}$ ). If the laser bandwidth $\Delta \omega$ is properly matched to the energy-level sep- aration of the molecular Hamiltonian, a close to "minimum uncertainty" wave packet can be produced, which moves very much like an ensemble of classical particles at short times.

Whether the wave packet can be approximated semiclassically, or whether it is thoroughly quantal in nature, its motion, which is a reflection of its coherence properties, can be imaged by a second laser pulse onto a higher electronic state (here the ion-pair $f$ state of $\mathrm{I}_{2}$ ). ${ }^{9}$ From a spectral perspective, the phases of the Franck-Condon factors (FC, including in this discussion a time-dependent phase) for the probe transition can interfere constructively or destructively, depending on the location of the wave packet on the intermediate surface. Of course, this does not imply that pumping with ultrashort pulses can create a strong transition in a wavelength region where none existed in a cw experiment, simply by shifting the wave packet to a better position, but the FC factors can interfere to yield transitions several times stronger or weaker (depending on the number of levels involved in the coherence) than the cw average, and oscillating about that average as a function of pump-probe delay. Note also in Fig. 1 that the pump as well as probe transitions tend to conserve the kinetic energy of the wave packet, at least in the semiclassical limit under resonant-transition conditions (extended FC principle for wave packets).

The coherence in the intermediate state is thus probed as a function of the time delay between pump and probe pulses. In practice; this is accomplished by scanning one of the pulses on a calibrated translation stage. As seen in Sec. IV, this calibration, rather than the temporal length of the coherent transient, can be the factor limiting accuracy. The typc of transicnt obtaincd in the specific case of $I_{2}$ is shown in Fig. 2(a). The vibrational motion of the wave packet yields rapid oscillations at early times, which are damped because the vibrational frequencies are not commensurate, and because the rotation-vibration coupling shifts the vibrational frequencies as the rotational excitation increases. At long times, the rotating dipoles realign (if polarized laser excitation was used), with a period determined by the rotational constants in the various vibrational levels populated by the pump pulse purc rotational coherencc. ${ }^{10}$

After the probe pulse, the population in the final state can be detected as cw fluorescence, ${ }^{11}$ a multiphoton ionization (MPI) signal, ${ }^{12}$ or by chemical reaction means. ${ }^{13}$ The partial coherence thus loses nearly all information about the initial or final electronic states, since only the coherence in the intermediate state is probed at different times by the second laser pulse. This can be an advantage, since it allows one to do spectroscopy on a single potential function (or surface, PES, in general) at a time. Furthcrmore, as discussed in Sec. IV, it makes the determination of molecular parameters independent of the laser wavelengths and profiles, which is desirable, since these quantities need then not be accurately determined. The coherence preserves all the relative spectral information (e.g., vibrational frequency differences) of the intermediate state, which is sufficient to characterize that state (except for its term energy). 
(A)

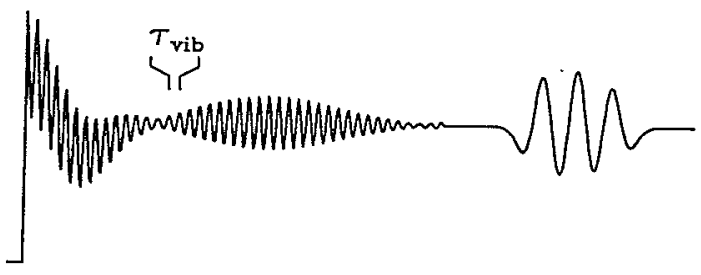

(B)

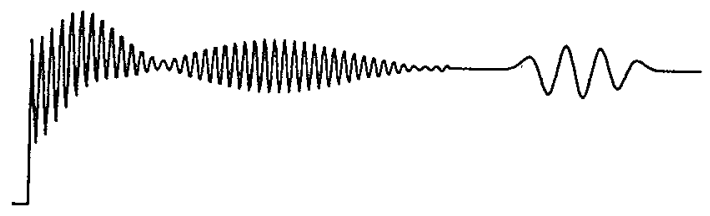

(C)

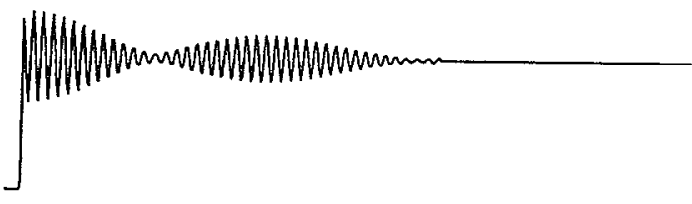

(D)

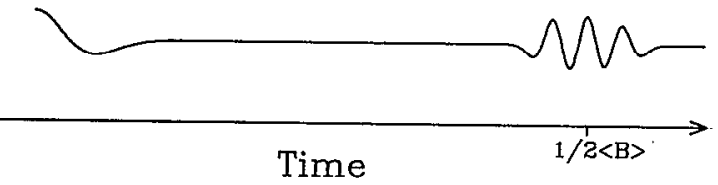

FIG. 2. Separation of rotations and vibrations by time scale and polarized detection; (A) shows a transient obtained in parallel detection, (B) one in perpendicular detection; (C) corresponds to magic angle detection, which removes both early time and later time rotational anisotropies; (D) corresponds to anisotropic detection (parallel minus perpendicular), showing only the rotational effects; as discussed in the text, rotation-vibration couplings still affect the envelope of transient (C) even in magic-angle detection.

Thus if one were to determine the power spectrum of the vibrational transient 2(c), it might appear as shown in Fig. 3; a cluster of lines near the fundamental vibrational spacing $\omega$, another weaker cluster at $2 \omega$ (weaker due to the chosen finite spectral bandwidth of the laser and rotationvibration couplings), a weaker one still at $3 \omega$, and so forth. It is clear that these peaks should correspond to vibrational frequencies in the intermediate electronic state, but it remains to be seen in Secs. III C and IV how precise vibrational constants can be extracted from the transient in Fig. 2 (c).

The incoherent effects of the initial ensemble can be almost eliminated by using a molecular beam, where a minimal number of rotational and vibrational levels of the ground state are populated. ${ }^{12}$ Such cooling is usually advantageous in high resolution spectroscopy, where collimated molecular beams are used to reduce spectral congestion and Doppler broadening. As will be seen below when comparing cell and molecular beam results, femtosecond wave packet spectroscopy (FWS) actually works best at higher temperatures, where it extracts the most information, especially for small systems. By its nature as a coherent rather than a spectral experiment on a time scale

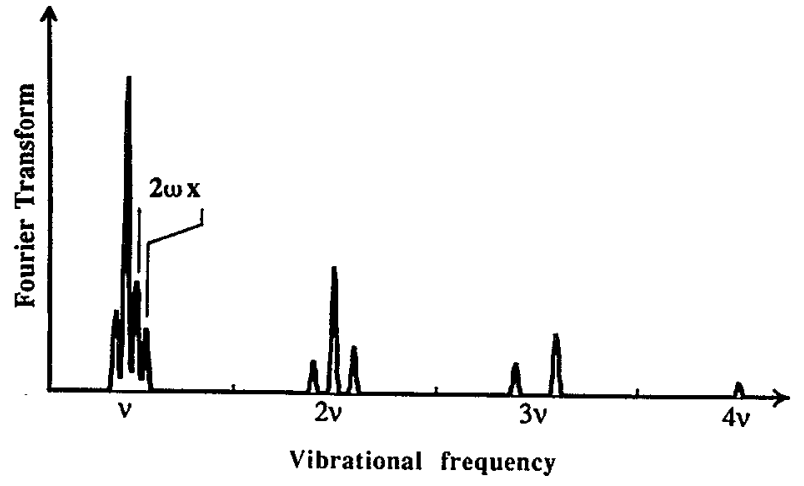

FIG. 3. Amplitude spectrum of the vibrational transient in Fig. 2(c); in $I_{2}$, there is a cluster at the local vibrational frequency, corresponding closely to the several vibrational band origins covered by the laser profile; at higher frequencies, the cluster is repeated with successively fewer lines, corresponding to energy differences between levels separated by $\Delta v$ $=2,3 \cdots$.

smaller than any molecular collisions, it removes the problem of Doppler broadening. For example, rotational constants can be read off directly from the recurrence time, and determined to some fractional accuracy, even when they are very small (below the resolution of a cw experiment); also, the molecular frequencies contained in a rovibrational coherence are unchanged, since the Doppler effect only shifts the laser frequency and bandwidth in the molccular frame, causing a slightly different set of energy levels to be overlapped.

A further consequence of this spectroscopy is a separation of time scales, and a convenient separation of scalar and vector effects in the molecular motion, as mentioned above. Vibrations generally occur on a femtosecond time scale, while rotations occur on a time scale of 10 to 1000 ps, depending on the force constants, geometries, and masses involved. Spin-orbit splittings and other effects may occur on even longer time scales, from 1 to $1000 \mathrm{~ns}$. As shown in Fig. 2(a), the rotational and vibrational time scales in $\mathrm{I}_{2}$ are indeed very different. Furthermore, polarized detection can eliminate either scalar (e.g., vibrational) or vector (e.g., rotational) effects [Figs. 2(b)-2(d)], depending on the angle between the two laser pulse electric fields (and the detector). ${ }^{1}$

As discussed below, this does not, of course, eliminate the rotation-vibration interactions inherent in the molecular Hamiltonian, which must still be considered in a detailed analysis. The most obvious manifestation in Fig. 2 (a) is the fact that, while the rotational constant can be seen to be approximately $1 / 2 t_{R}\left(t_{R}\right.$ being the time for a full rotational recurrence), there are several peaks corresponding to the several vibrational states present in the superposition (and also the centrifugal distortion interaction, as discussed in Ref 5). However, it will be seen below that the vibrational (early time) and rotational (long time) transients can always be treated scparately from one another, in effect decoupling the simultaneously obtained rotational and vibrational information. 


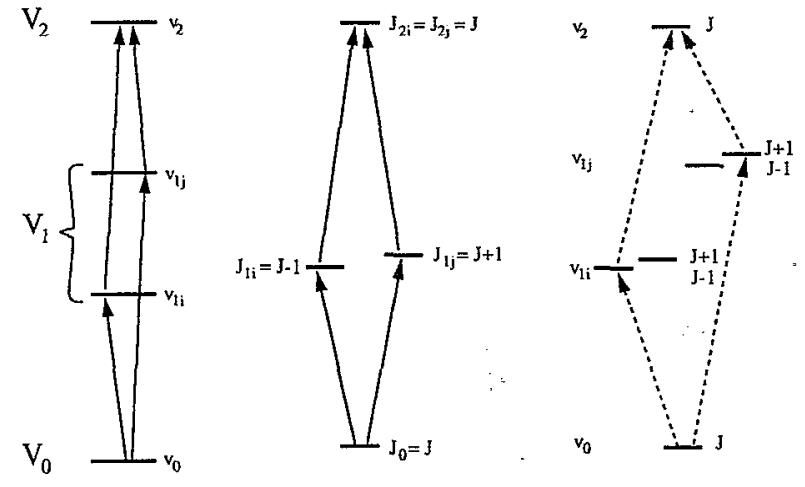

FIG. 4. Schematic representation of two-level quantum interference; (left) vibrational; (middle) rotational; (right) 2 by 2 level rotationvibrational; $V_{0}, V_{1}$, and $V_{2}$ refer to the electronic potential surfaces on which the levels lie, $v_{0}, v_{1}, v_{2}$ are the corresponding vibrational quantum numbers.

\section{COHERENCES: VIBRATIONAL AND ROTATIONAL}

The general formalism of quantum coherence relevant to the present work has been discussed in a number of articles (see, e.g., Refs. 10, 14, and 15). Here, we consider first a simple picture, and then a more general formulation. A number of simplifications are made, which will elucidate the underlying physics and provide a more effective framework for analyzing actual experimental data by highlighting the important features.

\section{A. Simple picture}

Consider Fig. 4(a), for the case of a vibrational twolevel system $\left(\left|v_{1 i}\right\rangle\right.$ and $\left|v_{1 j}\right\rangle$ on surface $\left.V_{1}\right)$ accessed by a pump pulse from a single vibrational level $\left|v_{0}\right\rangle$. After the pulse, the part of the state of interest evolves as

$$
\begin{aligned}
\left|\Psi_{1}\right\rangle \sim a_{01}\left(v_{1 i}\right) e^{-i 2 \pi c E_{1}\left(v_{1 i}\right) \tau}\left|v_{1 i}\right\rangle & \\
& +a_{01}\left(v_{1 j}\right) e^{-i 2 \pi c E_{1}\left(v_{1 j}\right) \tau}\left|v_{1 j}\right\rangle,
\end{aligned}
$$

where energies and frequencies are in $\mathrm{cm}^{-1}$. We are excluding here coherences between the $V_{0}$ and $V_{1}$ surfaces, which are possible in principle, but not probed in the present experiments. (With a stable-enough interferometer, this may be possible.) The factors $a_{01}$ depend on the laser electric field and transition dipole moment, $a_{01} \propto G_{L 01} \mu_{01}$.

In order for a coherence to be observed, the probe pulse must excite both components of the wave function to the same state $\left|v_{2}\right\rangle$, which may be detected by LIF, for example. Again neglecting coherences between $V_{2}$ and $V_{1}$, we obtain for the final signal

$$
\begin{aligned}
I(t) \sim & \left|\left\langle v_{2}\left|G_{L 12} \mu_{12}\right| \Psi_{1}\right\rangle\right|^{2} \\
\propto & a_{01}\left(v_{1 i}\right)^{2} a_{12}\left(v_{1 i}\right)^{2}+a_{01}\left(v_{1 j}\right)^{2} a_{12}\left(v_{1 j}\right)^{2} \\
& +2 a_{01}\left(v_{1 i}\right) a_{12}\left(v_{1 i}\right) a_{01}\left(v_{1 j}\right) a_{12}\left(v_{1 j}\right) \\
& \times \cos \left(2 \pi c\left[E_{1}\left(v_{1 j}\right)-E_{1}\left(v_{1 i}\right)\right] \tau\right) \\
= & c_{i}^{2}+c_{j}^{2}+2 c_{i} c_{j} \cos \left(2 \pi c\left[E_{1}\left(v_{1 j}\right)-E_{1}\left(v_{1 i}\right)\right] \tau\right),
\end{aligned}
$$

which contains oscillations due to coherence in $V_{1}$. Coherence effects due to $V_{0}$ and $V_{2}$ are eliminated since phase incoherent pulses and time-independent detection of the final state are used, yielding a spectroscopy with frequency components sensitive to only the intermediate surface. The above results are reminiscent of those obtained in the studies of $\mathrm{IVR}^{2}$ and highlight the nature of the phase shift, depending on the detection scheme, and the modulation depth, depending on the $c$ 's.

Collecting the $c$ 's in a constant $A_{V i j}$, it is easily seen that for more levels, one obtains for the oscillatory part

$$
I_{V}(t) \sim 2 \sum_{i j} A_{V i j} \cos \left(2 \pi c\left[E_{1}\left(v_{1 i}\right)-E_{1}\left(v_{1 j}\right)\right] \tau\right)
$$

A similar response is found for two rotational levels, 2,10 as shown in Fig. 4(b). Here the application of the vector dipole model yields $J$-dependent amplitude factors. For example, for parallel transitions (diatomic or linear molecule) with selection rules $\Delta J= \pm 1$ and two laser pulses with parallel relative polarizations, the cross term analogous to Eq. (2) becomes

$$
I_{R}(t) \sim \frac{2}{15} \frac{J(J+1)}{2 J+1} \cos (2 \pi c[E(J+1)-E(J-1)] \tau),
$$

including the $M$-level degencracy of the $\left|J_{0}=J\right\rangle$ initial state. The generalization to a sum over a large number of $J$ levels, usually weighted by a Boltzmann factor, is again clear.

In the most general case, cross terms between rotation and vibration must be considered, as in the $2 \times 2$ rovibrational level system in Fig. 4(c). For each vibrational coherence $v_{1} / v_{1}+1$, there are now four rotational terms contributing, namely, $J+1 / J+1, J-1 / J-1, J+1 / J-1$, and $J-1 / J+1$ [the last shown in Fig. 4(c)]. The first two contain no rotational anisotropy (but do contain vibration-rotation interaction, since they are still $J$ dependent), and do not cancel in magic-angle detection; the latter two result in rotational anisotropy, and cancel in magic angle detection.

\section{B. General picture}

For generalization we shall use the density matrix approach, invoked before in the description of rotational and vibrational coherence on the picosecond time scale..$^{2,10,14,15}$ For femtosecond pump-probe methodology, Mukamel has shown the power of the method in describing observables and Lin and Mathies have developed applications to temporal and spectral studies. ${ }^{16}$

Since the final detection step, whether optical, mass analyzed, or chemical, does not preserve any coherence of the final (third) electronic state of the pump-probe sequence, the detected signal is proportional to a weighted sum of the populations in each of the final eigenstates. Consider LIF detection as an example (Fig. 1). Emission takes place to a fourth state (or set of states), and the weighting factor $O\left(q_{2}\right)$ (with appropriate units) for a single state in $V_{2}$ is a convolution of all its transition strengths 
for emission with a wavelength-dependent response function of the detection system. This corresponds to a weighted trace over the density matrix describing the final state,

$$
I(\tau)=\operatorname{Tr}_{\left\{q_{2}\right\}}\left[O\left(q_{2}\right) \rho(\tau)\right]
$$

where $\left\{q_{2}\right\}$ indicates a trace over all quantum numbers describing the system after the probe pulse (e.g., $v_{2}, J_{2}$, and $M_{2}$ quantum numbers in the $f$ state of $\mathrm{I}_{2}$ ), and $\tau$ is the time lag between the pump and probe pulses which yields the time-dependent coherent transient $I(\tau)$.

One obtains for $\rho(\tau)$, using the standard density matrix formalism, the symmetrical expression

$$
\begin{aligned}
& \left.\rho(\tau) \sim \sum_{\substack{v_{2 i} J_{2 i} M_{2 i} \\
v_{2 j} J_{2 j} M_{2 j}}} \sum_{\substack{v_{1 i} j_{1} M_{1 i} \\
v_{1} J_{1 j} M_{1 j}}} \sum_{v_{0} J_{0} M_{0}}\left|J_{2 i} M_{2 i}\right\rangle \mid J_{2 i} v_{2 i}\right) e^{-i 2 \pi c E_{2}\left(v_{2 i} J_{2 i}\right) r+\phi_{12}} \times G_{L 12}\left[E_{2}\left(v_{2 i}, J_{2 i}\right)-E_{1}\left(v_{1 i} J_{1 i}\right)-\omega_{12}\right] \\
& \times\left\langle J_{2 i} M_{2 t}\left|\hat{z} \cdot\left\{\begin{array}{l}
\hat{Z} \\
\hat{X}
\end{array}\right\}\right| J_{1 t} M_{1 i}\right\rangle\left\langle J_{2 t} \nu_{2 t}\left|\mu_{12}(r)\right| J_{1 i} v_{1 i}\right\rangle \omega_{12} e^{-i 2 \pi c E_{1}\left(v_{1 i} J_{1 i}\right) \tau+\phi_{01}} \\
& \times G_{L 01}\left[E_{1}\left(v_{1 i}, J_{1 i}\right)-E_{0}\left(v_{0}, J_{0}\right)-\omega_{01}\right]\left\langle J_{1 i} M_{1 i}\left|\dot{z} \cdot\left\{\begin{array}{l}
\hat{Z} \\
\hat{X}
\end{array}\right\}\right| J_{0} M_{0}\right\rangle\left\langle J_{1 i} v_{1 i}\left|\mu_{01}(r)\right| J_{0} v_{0}\right\rangle \omega_{01} g_{n}^{1 / 2}\left(J_{0}\right) \\
& \times e^{-h c E_{0}\left(v_{0}, J_{0}\right) / k T} g_{n}^{1 / 2}\left(J_{0}\right) \omega_{01}\left\langle J_{0} v_{0}\left|\mu_{01}(r)\right| J_{1 j} v_{1 j}\right\rangle \times \cdots \quad \cdots \times\left\langle J_{2 j} v_{2 j}\right|\left\langle J_{2 j} M_{2 j}\right| .
\end{aligned}
$$

The $\cdots$ indicates the second half of the symmetric density matrix expression. $|J M\rangle$ are the standard rotational basis functions, $\left|J_{n} v_{n}\right\rangle$ are the eigenstates of the adiabatic diatomic rovibrational Hamiltonian

$$
H_{n}(r)=\frac{P_{r}^{2}}{2 \mu}+\frac{J_{n i}\left(J_{n i}+1\right) \hbar^{2}}{2 \mu r^{2}}+V_{n}(r)
$$

with eigenvalues $E_{n}$. Consider Eq. (6) in light of a pump-probe sequence, from the center (fourth line) up: An incoherent ensemble, here given by a Boltzmann distribution, with nuclear spin degeneracy $g_{n}\left(J_{0}\right)$, is pumped by a laser beam centered at $\omega_{01}$. To each transition from $V_{0}$ to $V_{1}$ corresponds a dipole vector operator $\left[\mu_{01}(r) \hat{z} \cdot\{\hat{z}\}\right.$, depending on the laser polarization in the $\hat{Z}$ or $\hat{X}$ direction] matrix element and a laser electric field strength $\left(G_{L 01}\right.$, depending on the offset of the transition frequency from the center frequency $\omega_{01}$ ). The state then freely propagates for a time $\tau$, after accumulating a phase factor $\phi_{01}$ during the pump process, which depends on the laser pulse characteristics, and may vary for different eigenstates if the pulse is not transform limited. The same procedure is repeated with a probe pulse, finally yielding a partially coherent superposition of states $\left|v_{2} J_{2} M_{2}\right\rangle \approx\left|J_{2} M_{2}\right\rangle\left|J_{2} v_{2}\right\rangle$ on $V_{2}$. The subscript $i$ indicates columns, the subscript $j$ rows of the density matrix.

Depending on the type of electronic transitions and the laser polarization, the above expression can be greatly simplified. In the case of two parallel electronic transitions probed by laser pulses of the same polarization, $\Delta J= \pm 1$, $\Delta M=0$. If we also neglect all off-diagonal components of $\rho$, since they do not contribute to the final trace, and set $t=0$, since the detection process is incoherent and the phase on surface 2 does not contribute,

$$
\begin{aligned}
\rho^{\prime}(\tau) \sim & \sum \sum_{\text {all } v J}\left|J v_{2}\right\rangle\left\langle J v_{2}\right| e^{-i 2 \pi c\left[E_{1}\left(v_{1 j} J \pm 1\right)-E_{1}\left(v_{1 j} J \mp 1\right)\right] \tau+\Delta \phi_{01}} G_{L 12}\left[E_{2}\left(v_{2}, J\right) \cdots E_{1}\left(v_{1 i}, J \pm 1\right) \cdots \omega_{12}\right] G_{L 12}^{+}\left[E_{2}\left(v_{2}, J\right)\right. \\
& \left.-E_{1}\left(v_{1 j} J \mp 1\right)-\omega_{12}\right] G_{L 01}\left[E_{1}\left(v_{1 i}, J \pm 1\right)-E_{0}\left(v_{0}, J\right)-\omega_{01}\right] G_{L 01}^{+}\left[E_{1}\left(v_{1 j}, J \mp 1\right)-E_{0}\left(v_{0}, J\right)-\omega_{01}\right] \\
& \times \mu_{12}\left(v_{2}, v_{1 i}\right) \mu_{12}^{+}\left(v_{2}, v_{1 j}\right) \mu_{01}\left(v_{1 i}, v_{0}\right) \mu_{01}^{+}\left(v_{1 j}, v_{0}\right) \times g_{n}(J) \omega_{12}^{2} \omega_{01}^{2} e^{-h c E_{0}\left(v_{0}, J\right) / k T} \\
& \times \sum_{M}|J M\rangle\langle J M| J(J+1)\left(\begin{array}{ccc}
J_{ \pm} 1 & J & 1 \\
M & -M & 0
\end{array}\right)^{2}\left(\begin{array}{ccc}
J \mp 1 & J & 1 \\
M & -M & 0
\end{array}\right)^{2} .
\end{aligned}
$$

The first line shows the time dependence of the coherence itself, the end of the first and second lines weight the rovibrational transitions according to the strength of the laser pulses, the third line contains the contributions of the vibrational transition dipole moments, and the initial incoherent superposition and frequency dependence of the ab- sorption. The sum over $M$ simply becomes the standard $J$-dependent weight for a parallel two-photon transition in the absence of a perturbing field [e.g., the same factor as in Eq. (4) for the $J+1 / J-1$ or $J-1 / J+1$ cases]. In the case of two laser beams with perpendicular relative polarization, the last line in Eq. (8) must be replaced by 


$$
\begin{aligned}
& \sum_{M}|J M\rangle\langle J M| J(J+1) \\
& \quad \times\left(\begin{array}{ccc}
J \pm 1 & J & 1 \\
M & -M & 0
\end{array}\right)^{2}\left(\begin{array}{ccc}
J \mp 1 & J & 1 \\
M \pm 1 & -M \mp 1 & 0
\end{array}\right)^{2} .
\end{aligned}
$$

The $J \pm 1$ and $J \mp 1$ are summed independently of one another, contributing four terms to the density matrix, as discussed in the previous subsection. We may symbolically write $\rho^{\prime}(t)$ as

$$
\begin{aligned}
\rho^{\prime}(t)= & \sum f_{i}(J+1) f_{j}^{\dagger}(J+1)+\sum f_{i}(J-1) f_{j}^{\dagger}(J-1) \\
& +\sum f_{i}(J+1) f_{j}^{\dagger}(J-1)+\sum f_{i}(J-1) f_{j}^{\dagger}(J+1) .
\end{aligned}
$$

Two of the terms $(J+1 / J+1$ and $J-1 / J-1)$ contribute mainly to the vibrational coherence when $v_{1 i} \neq v{ }_{1 j}$, while the remaining ones $(J+1 / J-1$ and $J-1 / J+1)$ contribute to the rotational coherence when $v_{1 i}=v_{1 j}$ For example, the last two terms in the case of parallel relative laser polarizations and parallel transitions yield the factor in Eq. (8).

\section{Useful simplifications}

Expressions such as Eqs. (6) and (8), while rigorous, are not very practical when fitting large sets of data, nor do they yield physical insight into the factors contributing to the transients. At all but the lowest rotational temperatures, the vibrational and rotational contributions (the two pairs of two terms mentioned above) can be separated. Consider first a magic-angle vibrational transient: It may be obtained by proper summing of Eq. (8) (parallel polarization) with Eq. (9) (perpendicular polarization), canceling the $J+1 / J-1$ and $J-1 / J+1$ terms in Eq. (10). After taking the final trace (dropping unnecessary proportionality constants whenever possible, and the subscript "1" on $v$ since we henceforth consider coherences only on surface 1),

$$
\begin{aligned}
I_{v}(\tau) \sim & \sum_{J} g_{n} e^{-h c E_{0 R}(J) / k r_{R}} \sum_{i j<i} A_{V i j}\left[J / 3 \cos \left(2 \pi c \tau\left[E_{v}\left(v_{i}, J-1\right)-E_{v}\left(v_{j}, J-1\right)\right]+\Delta \varphi_{i j}\right)\right. \\
& \left.+\frac{J+1}{3} \cos \left(2 \pi c \tau\left[E_{v}\left(v_{i j}, J+1\right)-E_{v}\left(v_{j}, J+1\right)\right]+\Delta \varphi_{i j}\right)\right] \\
\approx & \sum_{J} g_{n} J e^{-h c E_{0 R}(J) / k T_{R}} \sum_{i} A_{V i j} \cos \left(2 \pi c \tau\left[E_{v}\left(v_{i}, J\right)-E_{v}\left(v_{j}, J\right)\right]+\Delta \varphi_{i j}\right)
\end{aligned}
$$

where for $I_{2}$,

$$
\begin{aligned}
E_{v}(v+1, J)-E_{v}(v, J) \\
=\omega_{e}-2 \omega_{e} x_{e}(v+1)+3 \omega_{e} y_{e}\left(v^{2}+2 v+13 / 12\right) \\
\quad-\alpha_{e} J(J+1)+\cdots
\end{aligned}
$$

and the coefficients $A_{V i j}=A_{V i j}\left[O\left(v_{2}, J\right), G_{L 01}, G_{L 12}, k T\right.$, $\left.\mu_{01}, \cdots\right]$ include all the information on the detection method, laser profiles, vibrational populations, and transition moments for each pair of rovibrational levels contributing to the transient. The term in Eq. (12) will generally have the largest contribution, particularly for pump and probe pulses with relatively small bandwidth, but $\Delta v=2$ and higher vibrational coherences can also contribute to the transient. It should be noted that there is a rotational contribution, proportional to $\alpha_{e}$ in the lowest order, which will be seen to have a noticeable effect on even the magic angle vibrational transient.

In parallel detection of a parallel/parallel pump-probe sequence, the rotational transient becomes $\left[v_{1 i}=v_{1 j}\right.$ terms in Eq. (8)]

$$
\begin{aligned}
I_{R}(\tau) \sim & \sum_{i J} A_{R i B_{n}} \frac{4 J(J+1)}{15(2 J+1)} e^{-h c E_{0 R}(J) / k T_{R}} \\
& \times \cos \left(2 \pi c \tau\left[E_{R}\left(J+1, v_{i}\right)-E_{R}\left(J-1, v_{i}\right)\right]\right),
\end{aligned}
$$

where for $\mathrm{I}_{2}, E_{R}(J, v)=B_{v} J(J+1)-D_{v}[J(J+1)]^{2}+\cdots$. The appearance of the short-time and long-time transients is as shown in Fig. 2, and has been discussed in our previous letter. ${ }^{5}$ As for the vibrations, the rotational amplitudes $A_{R i i}$ lump together the intensity, transition dipole and laser profile information, and can be fitted from the data.

It should be noted again that the different time scales and polarized detection almost completely uncouple the rotational and vibrational transients in Eqs. (11) and (13). The remaining effects are (a) in the rotational transient, several peaks appear due to the several vibrations populated in the intermediate electronic state and centrifugal distortion; this has been discussed in detail previously; (b) in the vibrational transient, the rotation-vibration constant $\alpha_{e}$ has a subtle effect on its envelope and phase: If one were to determine frequencies from the transient by calculating 
a power spectrum, this would manifest itself as a broadening and shift in intensity and position of the vibrational peaks, due to their rotational envelope. To see the effect in the time domain, the rotational sum in Eq. (11) (including only $\Delta v=1$ coherences) is approximated by the integral

$$
\begin{aligned}
I_{v}(\tau) \approx & \sum_{i} A_{V i, i+1} \int_{0}^{\infty} d j j e^{-h c B j^{2} / k T_{R}} \\
& \times \cos \left\{2 \pi c \tau\left[\omega_{e}-\alpha_{e} j^{2}-2 \omega_{e} x_{e}\left(v_{i}+1\right)+\cdots\right]+\Delta \varphi_{i}\right\} \\
\propto & \sum_{i} A_{V i, i+1}\left\{1+b^{2} \tau^{2}\right\}^{-1}\left(\operatorname { c o s } \left\{2 \pi c \tau \left[\omega_{e}\right.\right.\right. \\
& \left.\left.-2 \omega_{e} x_{e}\left(v_{i}+1\right)+\cdots\right]+\Delta \varphi_{i}\right\}+b \tau \\
& \left.\left.\times \sin \left\{2 \pi c \tau\left[\omega_{e}-2 \omega_{e} x_{e}\left(v_{i}+1\right)+\cdots\right]+\Delta \varphi_{i}\right\}\right]\right)
\end{aligned}
$$

where $b=2 \pi \alpha_{e} k T_{R} / B h$ is a temperature dependent scaling factor (here, $B$ is an average rotational constant for the $X$ state of $\mathrm{I}_{2}$ ). Equation (14) is an excellent approximation at higher temperatures. It can be seen that if we do not explicitly take rotation-vibration interaction into account by summing over $J$ as well as $v$, the vibrational coherence is no longer given by a sum of harmonics, but rather by a sum of damped harmonics that phase shift by $90^{\circ}$ on a time scale of $b^{-1}$ ( $\approx 40 \mathrm{ps}$ for $\mathrm{I}_{2}$ at room temperature). The main effect is a more rapid decrease in vibrational transient intensity than expected for $J=0$, corresponding to the broadened power spectrum due to the presence of many rotational transitions in a vibrational envelope. A purely vibrational analysis which neglected this fact would yield band origins shifted by approximately $\Delta \omega \approx-\alpha_{e} k T / B h c$, leading to a slight reduction in the calculated frequencies.

A similar approximate formula can be obtained for the shape of the rotational transients, and may be useful when $k T>B$, and when centrifugal distortion can be neglected. Taking a single vibrational level with rotational constant $B_{v}$ from Eq. (13),

$$
\begin{aligned}
I_{R}(\tau) & \propto \int_{0}^{\infty} d j \frac{2}{15} j e^{-h c B_{0} j^{2} / k T_{R}} \cos \left(4 \pi c \tau B_{v} j\right) \\
& \propto L_{1 / 2}^{-1 / 2}\left(b^{2} \tau^{2}\right) e^{-b^{\prime 2} \tau^{2}} \\
& \propto\left(1-b^{\prime 2} \tau^{2}\right) e^{-b^{\prime 2} \tau^{2}}
\end{aligned}
$$

is the rotational transient in parallel detection near time 0 or measured from the center of a full recurrence. The quadratic approximation to the generalized Laguerre function $L_{1 / 2}^{-1 / 2}$ is a good approximation wherever $I_{R}(\tau)$ substantially differs from zero. The scaling factor $b^{\prime}$ equals $4 \pi c B_{v}\left(k T_{R} / B_{0} h c\right)^{1 / 2}$.

\section{Conversion from the time to the frequency domain}

As discussed in Ref. 5, the best way of obtaining rotational constants is a direct fit to Eq. (13). This is due to the fact that centrifugal distortion cannot be neglected and results in a "ringing" pattern after the rotational peaks. Since the ultrafast pump pulse generally covers only a few vibrational levels at a time, producing only a few oscilla- tions in $I_{R}$, this method is reliable even when all coefficients $A_{R i i}$ are fitted individually.

Vibrational constants could be determined using Eqs. (11) or (14). At a first glance, a nonlinear fit to Eq. (11) or (14) seems impractical, unless the frequencies are known fairly accurately to begin with, due to the large number of parameters that must be fitted. A more straightforward way would be the determination of the power spectrum of the early time transient in Fig. 2(a). In two earlier letters, we have applied both FFT and maximum entropy algorithms to the determination of power spectra; $;^{5,6}$ before discussing application to $I_{2}$, we briefly apply them, as well as Prony's spectral method ${ }^{17}$ and nonlinear fitting, to a test model.

Figure 5(a) shows a plot of a synthetic transient generated from three harmonics, without damping or phase distortion, as well as the "exact" power spectrum (if the transient had been extended to infinite times), which is the information we in principle desire. Consider now the four models we have tested.

At a first glance, Prony's spectral method, which is designed to extract frequencies from a time series due to exponentially damped harmonics, appears like an ideal choice. ${ }^{17}$ When the frequencies to be extracted are close to one another, as is the case here, we found this to be true only for very high signal-to-noise ratios. At low $\mathrm{S} / \mathrm{N}$ ratios, Prony's method fails. The reason for this lies in the manner in which it attempts to decouple the harmonic amplitudes from the frequencies, determining the latter by first solving a linear set of equations, then determining the former with the frequencies held fixed in another linear least squares fit. In effect, Prony's method has a short temporal correlation length, but when two frequencies are close together, their distinguishability requires the recognition of very slow changes in the amplitude envelope of a transient. In practice, the method can be made to converge by a careful choice of the equations to be included in the least-squares fit, but is not suitable for an "automated" determination of frequencies.

Fast Fourier transform (FFT) and the maximum entropy method (MEM) fare considerably better. The power spectra and transients derived from them are shown in Fig. 5. The results are symptomatic of the performance of these techniques.

FFT assumes a periodic continuation of the function, expanded in harmonics which are multiples of a single fundamental frequency. This is clearly not a perfect model for $\mathrm{Eq}$. (11), since the frequency components are better described as the sum a constant fundamental frequency and a (nearly) linearly increasing offset, at least in the present case. The result is an unnecessary broadening of lines due to spectral leakage, as well as a resolution limited by the sampling frequency (Nyquist limit). On the other hand, the amplitudes of the components are quite well represented.

Burg's MEM (see Ref. 17) assumes a maximally ran- 

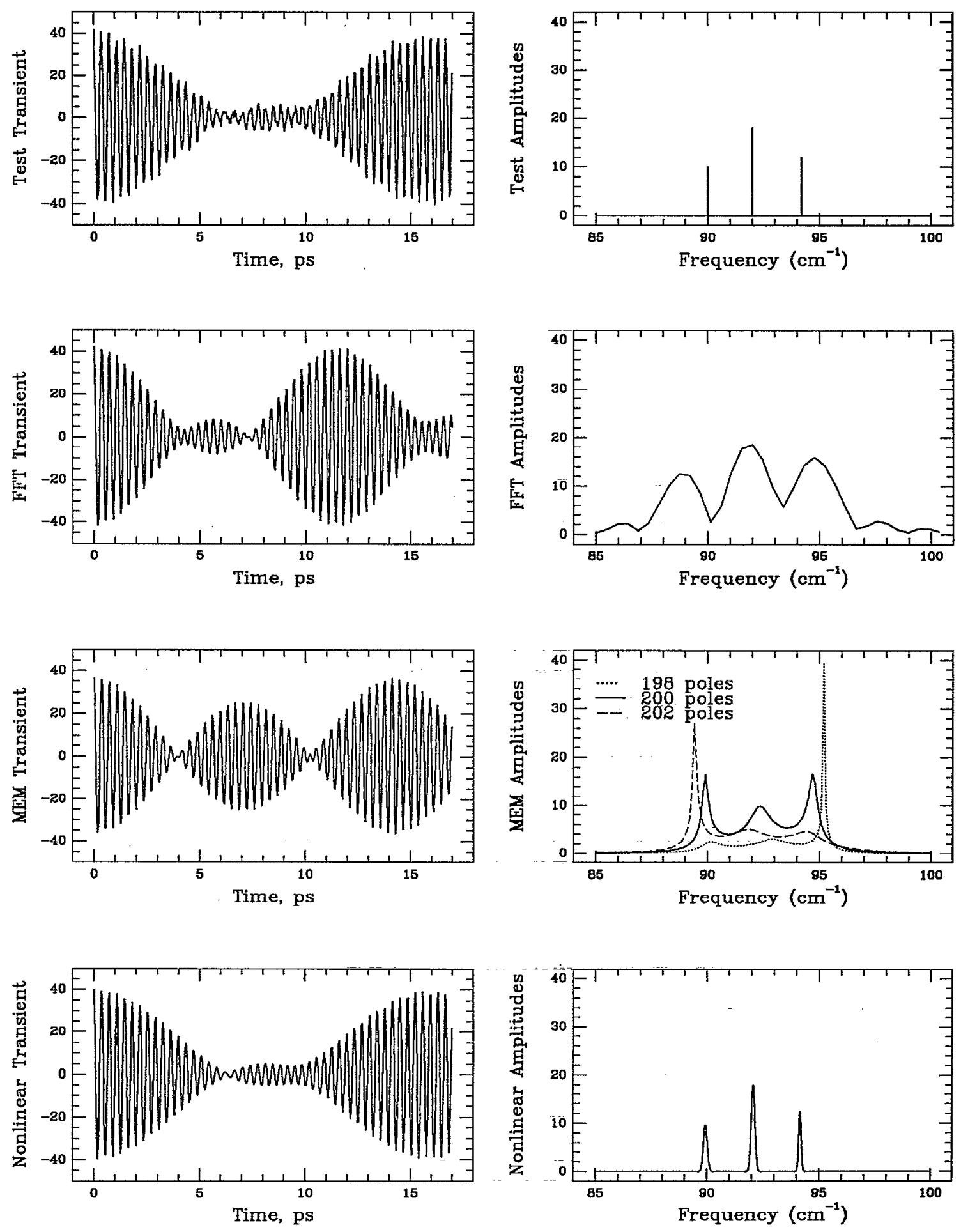

FIG. 5. Comparison of different methods for extracting frequency components from a temporal transient. The top row shows a test transient (no rotational effects) and the ideal power spectrum if the transient were noise free and extended to infinite times; the second row shows a fast Fourier amplitude spectrum and the transient generated from it; the third row shows a maximum entropy amplitude spectrum and its transient; note the sensitivity to pole number discussed in the text; the bottom row shows an amplitude spectrum extracted by the nonlinear fitting procedure described in the text, and the resulting temporal transient. The widths of the nonlinearly fitted peaks are the $2 \sigma$ precision of the nonlinear fit.

dom continuation of the function beyond the sampled region, representing the power spectrum by a denominator Padé expansion well suited for sharp peaks (due to the presence of poles in the analytic continuation of the Padé approximant). This model is also imperfect. In the case of noisy transients at nearby frequencies, it leads to highly inaccurate amplitudes, and fluctuations in the frequencies as a function of the number of poles included. In the case 
on hand, there is no "objective" reliable way of determining the number of poles in the expansion; as an approximate guide, the frequencies and peak amplitudes generally stabilize, while the peaks narrow, near the optimal number of poles (but the opposite may happen, at least if optimal is meant to indicate the smallest deviation from the correct frequencies); the resulting frequency rms error, $0.6 \mathrm{~cm}^{-1}$, is quite comparable to the error of the FFT spectrum, 0.75 $\mathrm{cm}^{-1}$ (close to the Nyquist step size).

For the present application, the general conclusions are as follows:

(1) FFT provides superior amplitudes;

(2) FFT and MEM provide comparable frequency accuracy with noisy spectra (MEM becomes increasingly better as the $\mathrm{S} / \mathrm{N}$ improves, since it is not hampered by the Nyquist limit);

(3) MEM provides superior peak identification, due to the narrower line widths.

Finally, we turn to nonlinear fitting methods. While it is not possible to nonlinearly fit a noisy transient directly, the following series of steps has been found to yield a very robust, reliable and automated means of determining frequency components without any a priori knowledge of their exact positions or amplitudes:

(1) Determine an approximate power spectrum by FFT or MEM;

(2) Use a peak finding routine to locate the strongest peaks;

(3) Fit the positions to a smooth model that approximately represents the system Hamiltonian (e.g., in the case of $\mathrm{I}_{2}, \omega_{i}=\omega_{0}+\Delta_{1} \omega i+\Delta_{2} \omega i^{2}$, where $i$ is an index offset from the vibrational quantum number by an unknown amount; if $i=0$ is chosen for the lowest frequency peak, $\omega_{0}$ represents the first vibrational frequency in the line cluster, and $\Delta_{1} \omega$ $\left.\approx-2 \omega_{e} x_{e}\right)$

(4) Fit intensities to a simple harmonic model by linear least squares: $I(t)=\Sigma A_{i} \cos \left(\omega_{i} t\right)+B_{i}$ $\times \sin \left(\omega_{i} t\right)$

(5) Relax all parameters in a nonlinear fit to a more accurate model, such as Eqs. (11) or (14).

The result of such a fit, implemented in a single program RECFIT, is shown in Figs. 5(g)-5(h), yielding by far the most precise frequencies and amplitudes, with a correspondingly longer processing time. The precision obtained by this method in the test case is $0.005 \mathrm{~cm}^{-1}$, or $10 \mathrm{ppm}$. The nonlinear fit model is thus capable of extracting frequencies with a precision well in excess of the Nyquist limit, with $\Delta v$ less than $1 / 2 t_{\mathrm{scan}}$ by 2 orders of magnitude in the best cases. It should be noted that this by no means represents a violation of the time-frequency uncertainty principle, which would dictate $\Delta v \sim 1 / \Delta t$ for measured frequency and temporal quantities: the frequencies reported here are fitted, not observed values, their precision being due to the choice of a fitting model better than FFT, and assumed $a$ priori to represent the data. Whether the frequencies are accurate in addition to being precise depends on the goodness of the model chosen, but since the Dunham Hamiltonian is known to rcpresent $I_{2}$ encrgy levcls to
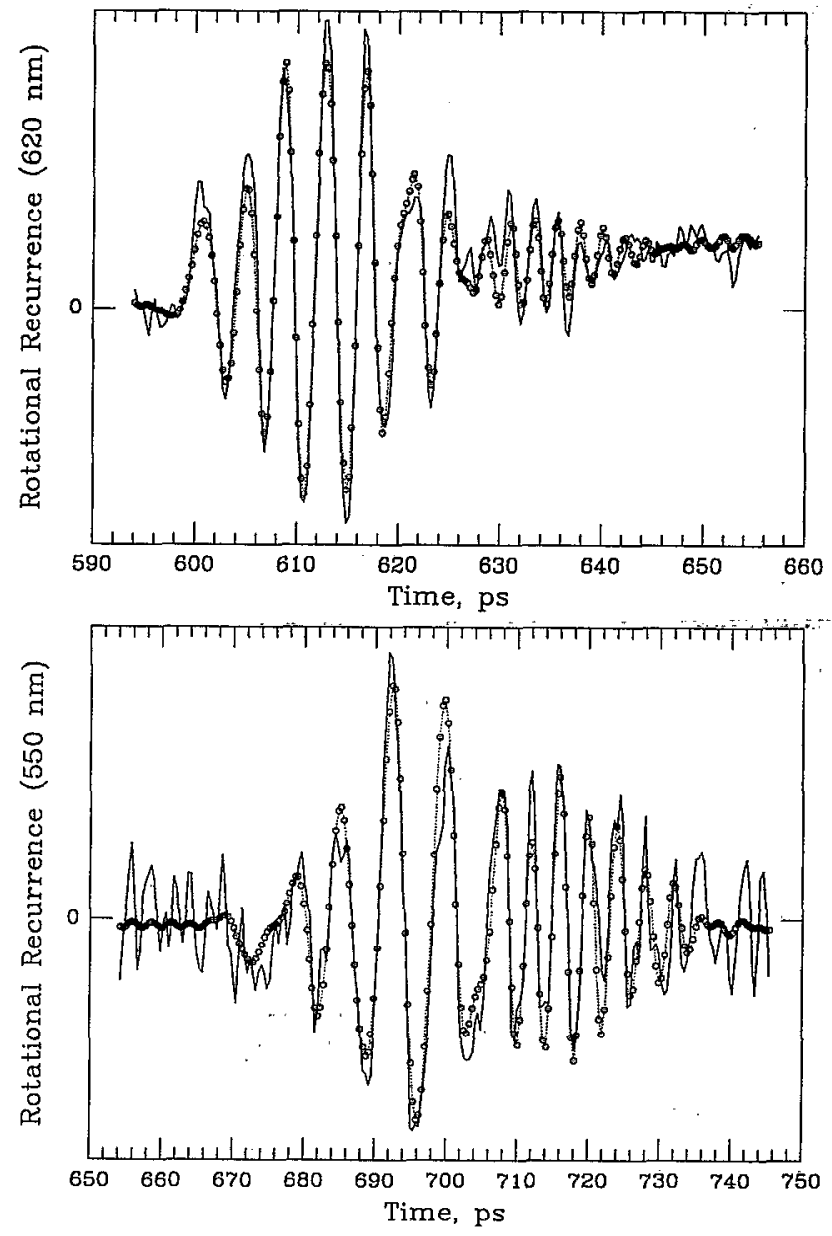

FIG. 6. Experimental full rotational recurrence at $620 \mathrm{~nm}(v \approx 9)$ and $550 \mathrm{~nm}(v \approx 25)$, detected with parallel laser polarization (solid line), and fit to Eq. (13) (dotted line and circles).

high accuracy, the frequencies obtained here will also be accurate. The analogy from high-resolution spectroscopy would be a determination of a band origin to better than the linewidth, by fitting an entire rovibrational band to a model Hamiltonian known to hold for the molecule under investigation. We will see in the following how these results carry over to real temporal data.

\section{APPLICATION: $\mathbf{I}_{2}$}

\section{A. Rotational transients}

We now consider the application to the rovibrational coherence of a real system, $I_{2}$ in the $B$ electronic state. Figure 6 shows as examples rotational transients obtained by pumping at $620 \mathrm{~nm}(v \sim 9)$, and $550 \mathrm{~nm}(v \sim 25)$, together with fits to Eq. (13). Inclusion of rotational intensity alternations and individual adjustment of vibrational level amplitudes has yielded a slight improvement over the fits in Ref. 5. The rotational constants for these particular levels are shown in Table I, together with precise highresolution values. The small differences, as well as methods for assigning vibrational quantum numbers, will be discussed together with the vibrational frequency determina- 
TABLE I. Comparison of FWS and accurate high resolution vibrational frequencies and rotational constants (in $\mathrm{cm}^{-1}$ ).

\begin{tabular}{|c|c|c|c|c|c|}
\hline \multicolumn{3}{|c|}{ Vibrational parameters } & \multirow[b]{2}{*}{ Frequency } & \multirow[b]{2}{*}{ Ref. 7} & \multirow[b]{2}{*}{ Ratio } \\
\hline Wavelength & $v l^{a}$ & $v u$ & & & \\
\hline \multirow[t]{5}{*}{$620 \mathrm{~nm}$} & 10 & 11 & 107.908 & 107.912 & 1.00004 \\
\hline & 9 & 10 & 109.598 & 109.631 & 1.00030 \\
\hline & 8 & 9 & 111.278 & 111.327 & 1.00044 \\
\hline & 7 & 8 & 112.957 & 113.002 & 1.00038 \\
\hline & 6 & 7 & 114.637 & 114.657 & 1.00017 \\
\hline \multirow[t]{4}{*}{$550 \mathrm{~nm}$} & 27 & 28 & 75.440 & 75.421 & 0.99975 \\
\hline & 26 & 27 & 77.500 & 77.483 & 0.99978 \\
\hline & 25 & 26 & 79.470 & 79.531 & 1.00077 \\
\hline & 24 & 25 & 81.430 & 81.563 & $\begin{array}{c}1.00163 \\
\langle 1.00040\rangle\end{array}$ \\
\hline
\end{tabular}

Rotational constants

\begin{tabular}{lccl} 
Wavelength & $v^{\mathrm{a}}$ & $B(v)$ & Ref. 7 \\
\hline $620 \mathrm{~nm}$ & 6 & 0.02797 & 0.02798 \\
& 7 & 0.02780 & 0.02781 \\
& 8 & 0.02762 & 0.02764 \\
& 9 & 0.02745 & 0.02746 \\
& 10 & 0.02727 & 0.02729 \\
$550 \mathrm{~nm}$ & 11 & 0.02708 & 0.02711 \\
& 23 & 0.02458 & 0.02462 \\
& 24 & 0.02434 & 0.02438 \\
& 25 & 0.02414 & 0.02414 \\
\hline
\end{tabular}

${ }^{a} v l$ and $v u$ are the vibrational levels assigned to a specific frequency component of a transient, $v$ is the vibrational quantum number assigned to a specific observed rotational recurrence peak.

tions. Here we just note that as before, centrifugal distortion (now up to third order) and a finite rotational temperature $(\sim 380 \mathrm{~K})$ had to be included to account for the observations.

This can be circumvented by the use of a molecular beam, but paradoxically, at a drastic loss in energy resolution. Figure 7 shows a rotational half-recurrence obtained

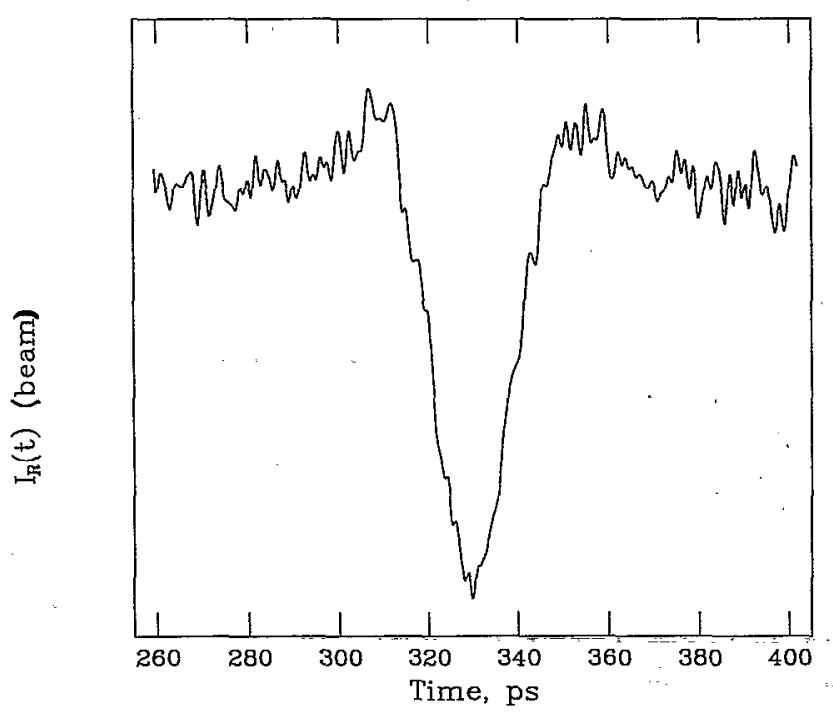

FIG. 7. Rotational half recurrence in a molecular beam [see M. Dantus, M. H. M. Janssen, and A. H. Zewail, Chem. Phys. Lett. 181, 281 (1991)].
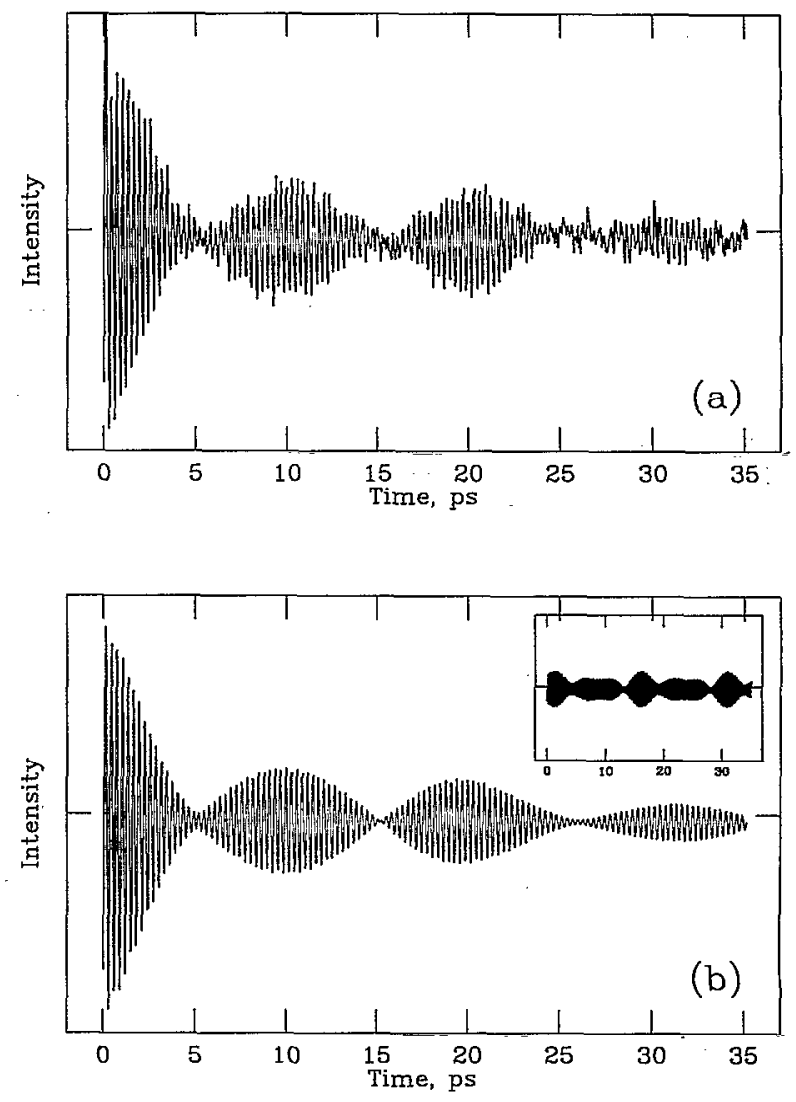

FIG. 8. (a) Magic-angle vibrational transient at a pump wavelength of $620 \mathrm{~nm}(v=9)$; (b) optimal fit to Eq. (14), with rotation-vibration interactions included in the amplitude damping and a nonlinear phase shift; the insert shows the best fit without taking rotation into account, i.e., to a sum of vibrational harmonics; six vibrational states were required to reproduce the experimental observations.

at $\lambda_{\text {pump }}=560 \mathrm{~nm}$. The rotational recurrence now has the simple appearance expected at low temperatures, ${ }^{10}$ without the "centrifugal distortion tail." Unfortunately, the width has increased to obscure the vibrational structure. Without knowing the laser bandwidth and temperature of the molecular beam, and at realistic signal-to-noise ratios, the number of vibrations covered and the $\alpha_{e}$ value cannot be properly decorrelated, making specific vibrational assignments impossible. As seen below, the vibrational frequencies are also better determined at higher temperatures, although for entirely different reasons.

\section{B. VIbrational transients}

Figure 8(a) shows an experimental magic angle vibrational coherence at $620 \mathrm{~nm}$ near room temperature $(v$ $\approx 23-27$ ). Figure 9 shows the corresponding power spectra obtained by the FFT, MEM, and nonlinear fitting methods. As in the test case discussed above, FFT and MEM power spectra (with optimally adjusted phase information added) cannot reproduce the experimental transient even in its gross features. Instead, Fig. 8(b) shows transients calculated from two different nonlinear fits. The insert assumes that $b=0$ in Eq. (14), i.e., neglects the rotation- 

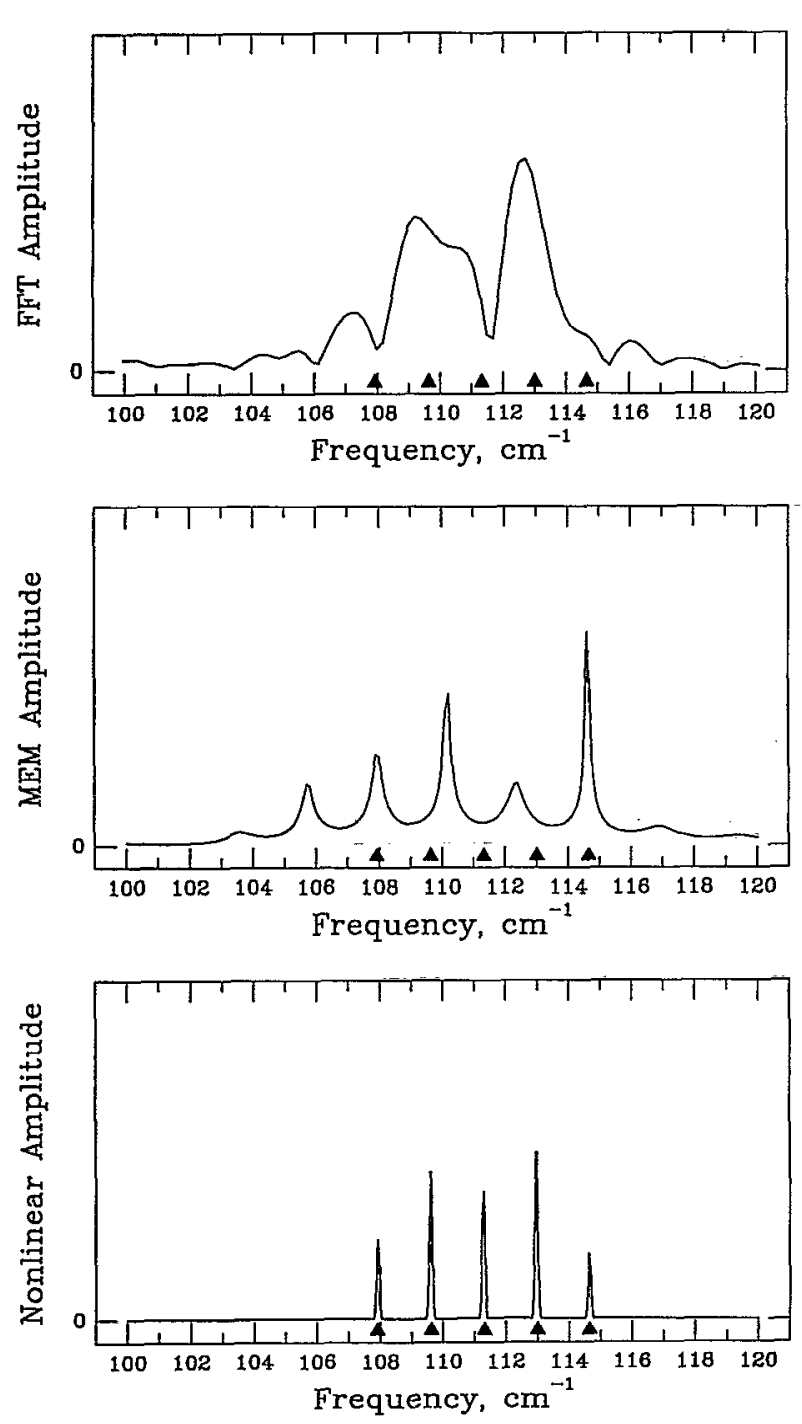

FIG. 9. Amplitude spectra corresponding to the $620 \mathrm{~nm}$ transient in Fig. 8; the top figure shows the FFT result (mean deviation $0.7 \mathrm{~cm}^{-1}$ ); the middle the MEM vibrational peaks (mean deviation $1.2 \mathrm{~cm}^{-1}$ ); the bottom the nonlinear fit results (mean deviation $0.02 \mathrm{~cm}^{-1}$ ); the actual vibrational frequencies, as obtained from $c w$ spectroscopy, are indicated by black triangles; as noted in the text, the broader peaks of the FFT do not necessarily indicate lower accuracy than obtained by the MEM method, and the FFT also reproduces the actual amplitudes somewhat better. The widths of the nonlinearly fitted peaks are the $2 \sigma$ precision of the nonlinear fit.

vibration interaction. The main plot shows the result of freely varying $b$, and reproduces the observed transient within the experimental noise, yielding a value of $b$ close to that expected. The vibrational frequencies obtained from the two fits agree to about $0.01 \mathrm{~cm}^{-1}$. Note that for simplicity's sake, we show the calculated vibrational frequencies of the levels needed to converge the nonlinear fit, rather than the local values of $\omega_{0}, \Delta_{1} \omega, \Delta_{2} \omega$, etc., fitted as described in the previous section. [See the following section on assignment of quantum numbers and how to obtain $\omega(v)$ from $\omega_{0}, \Delta_{1} \omega$, etc.] At most two anharmonic con-
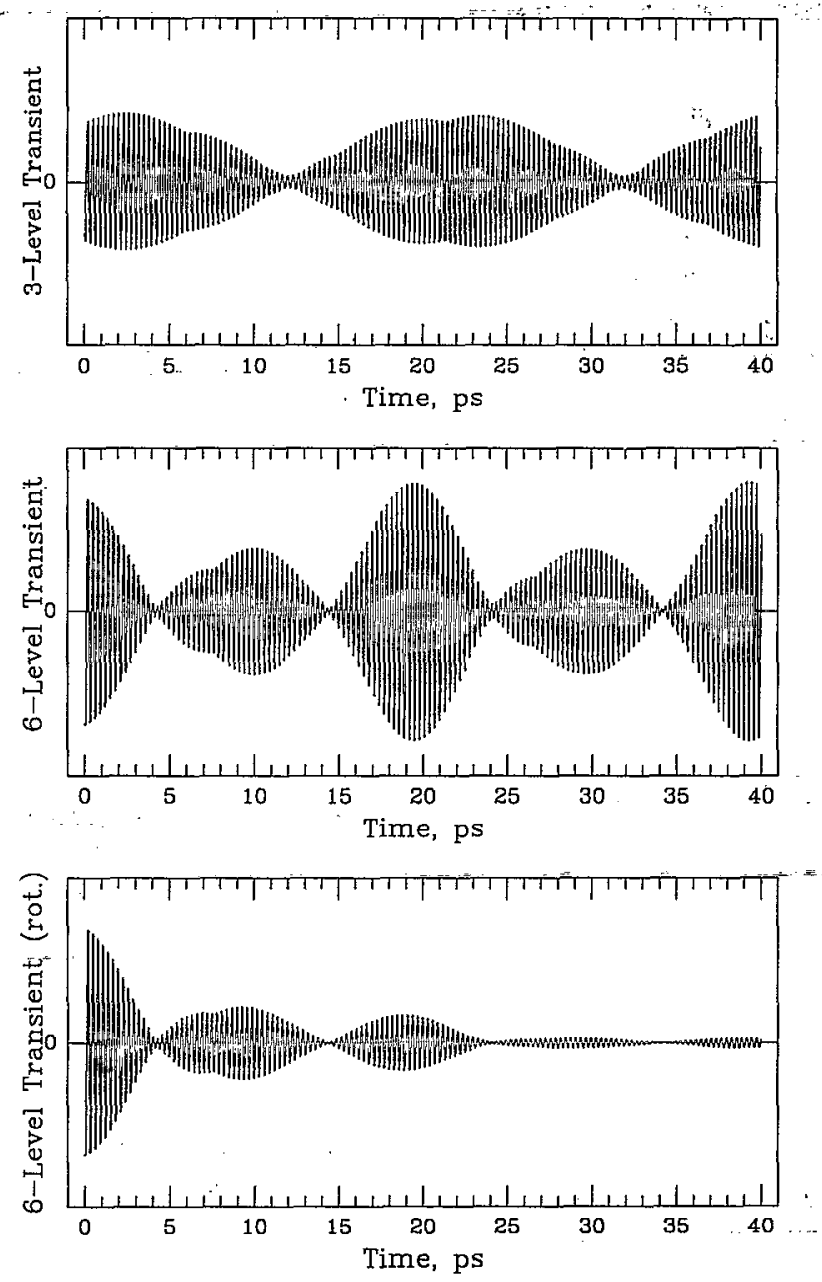

FIG. 10. Effect of multilevel interference on the transient; the top figure shows a threc-level transient ncar $v=9$; the rccurrence occurs at $t=1$ / $(2 c \omega x)$, as expected; the middle figure shows the full six-level transient, without rotational effect; a vibrational half-recurrence is observed due to intensity alternation of the vibrational amplitudes; the bottom shows the same transient including the rotational damping effect.

stants (up to $\Delta_{2} \omega$ ) were required to satisfactorily reproduce any of our transients.

One interesting feature in the $620 \mathrm{~nm}$ vibrational transient is the partial recurrence at $10 \mathrm{ps}$. This is only one-half the time expected from a simple anharmonic three-level picture of $I_{2}$ at $v=25$. Figure 10 shows three partial simulations of the $620 \mathrm{~nm}$ transient: At the top, only the three strongest amplitudes from the fit to Eq. (14) are included, and a full vibrational recurrence is observed at 20 ps. If three more vibrational amplitudes are added, the middle transicnt is obtaincd, which shows a strong partial vibrational recurrence, due to alternating vibrational amplitudes (due to FC factors). At the bottom of Fig. 10, the rotation-vibration interaction has been included, and the transient now strongly resembles the exact result of Fig. 8. Clearly, the maxima in the transient cannot always be straightforwardly "inverted" to the anharmonicity of the vibrational levels.

Another cell transient obtained at $550 \mathrm{~nm}(v \approx 25)$ and the corresponding best fit are shown in Fig. 11, yielding 

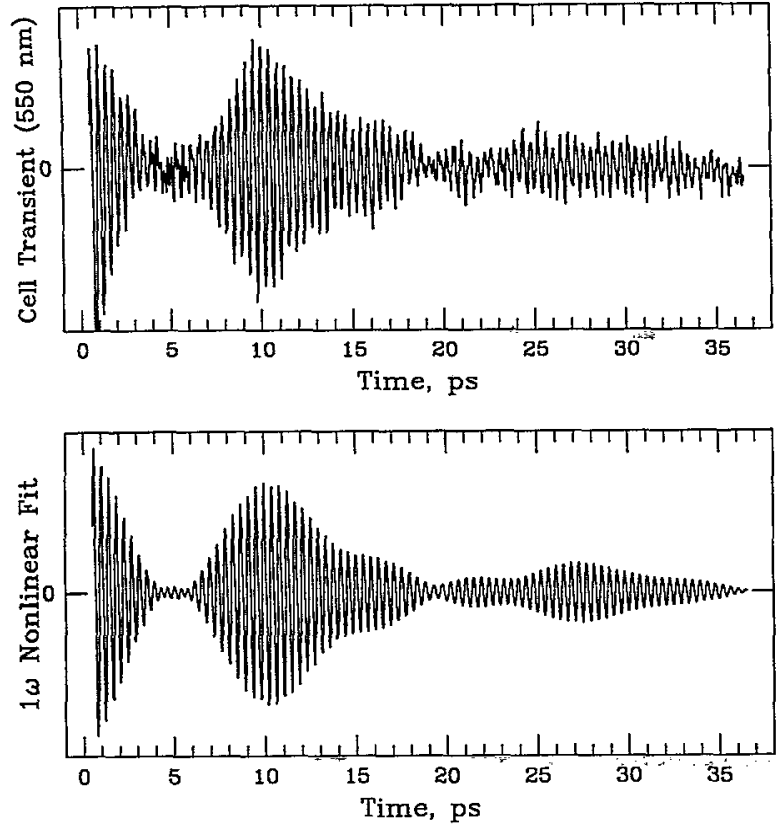

FIG. 11. Vibrational transient at $550 \mathrm{~nm}$ and room temperature; the nonlinear fit very nearly reproduces the experimental data, although only nearest-neighbor vibrational coherences are included, just as in Fig. 9.

similarly good agreement. Here the fit is to a parallel laser polarization vibrational transient, with the rotational anisotropy removed by fitting and subtraction.

The vibrational frequencies from the best fit are shown in Table I for the two transients. A conversion factor of $0.3951 \mathrm{fs} / \mathrm{step}$ was used in converting the motion of the translation stage used in the experiments from the distance to the time domain. Comparing to the fractional error with exact high-resolution measurements, it can be seen that the fitted vibrational frequencies show a small systematic deviation. Indeed, the deviation is smaller than one might expect from the calibration error. Since it appears in a number of entirely separate transients, it is probably safe to assume that the residual error of $0.04 \%$ is due to calibration. The accuracy in Table I, $0.03 \mathrm{~cm}^{-1}$, could thus be improved to $0.01 \mathrm{~cm}^{-1}$ with an interferometrically calibrated translation stage, yielding an accuracy comparable to $\mathrm{cw}$ experiments, and 10-50 times better than expected from Fourier transform techniques $\left(1 / 2 t_{\mathrm{scan}} \approx 0.6 \mathrm{~cm}^{-1}\right)$. The fairly good agreement of the $550 \mathrm{~nm}$ data further demonstrates that nearly the same accuracy can be obtained from artificial magic-angle transients as from true magic angle scans. In general, we find that the nonlinear fitting method outperforms the Nyquist limit by a factor of typically 10 or more for the best signal-to-noise ratio scans ( $\mathrm{S} / \mathrm{N}>15$ ), and reduces to performing near the Nyquist limit for transients with a signal-to-noise ratio below 2:1.

Fits to Eq. (11) instead of Eq. (14) produce very similar results, but require more computer time, due to the extended rotational summations needed at higher temperatures. We also considered fits including $(v+1 / 2)^{2} J(J$ $+1)$ and $(v+1 / 2) J^{2}(J+1)^{2}$ rotation-vibration terms in
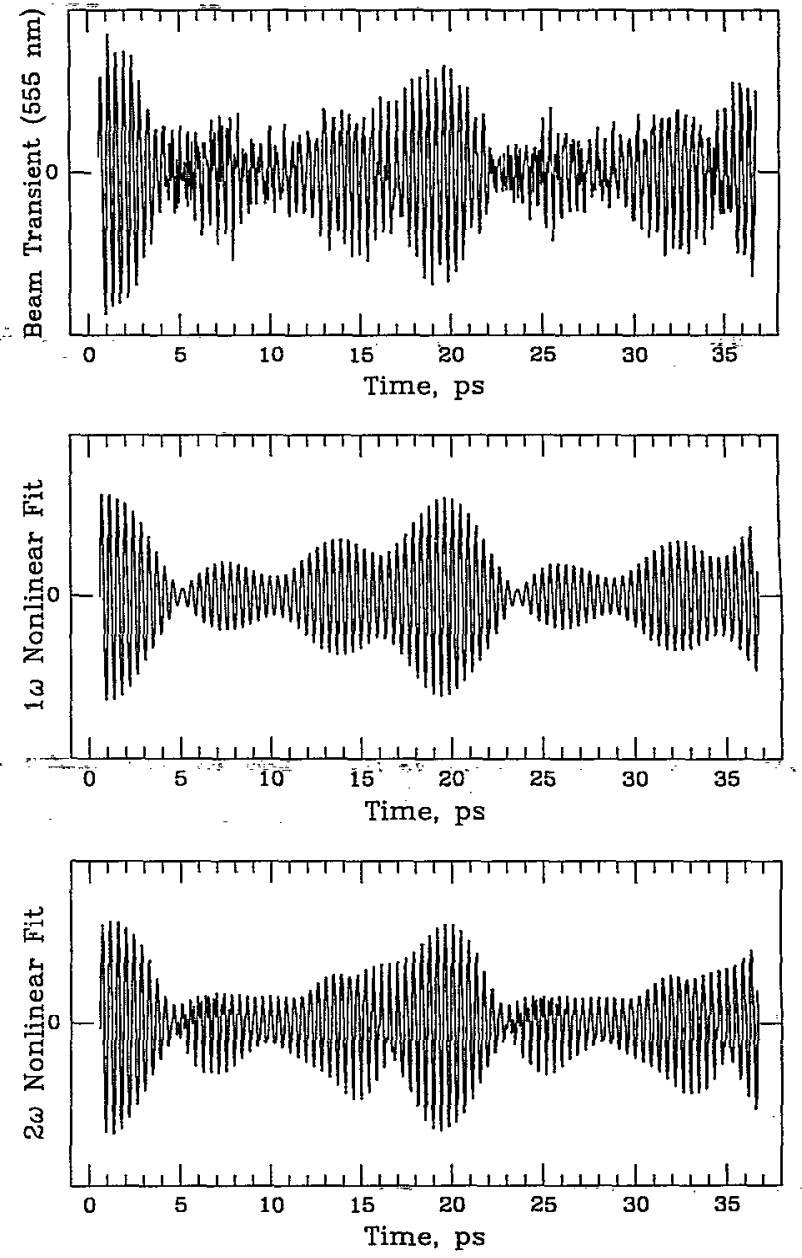

FIG. 12. Top: molecular beam transient at $555 \mathrm{~nm}$; middle: nonlinear fit, including only nearest-neighbor vibrational coherences; it has the same general envelope, but fails to account for higher frequency beat and a pronounced asymmetry of the transient; bottom: nonlinear fit, including also next-nearest-neighbor interactions; the asymmetric envelope scen in the experiment is reproduced, as well as the most pronounced higher frequency beats.

addition to the $(v+1 / 2) J(J+1)$ term, but found them to yield no significant improvement. Even at higher temperatures, their effect on the frequencies should remain small, as even letting $b=0$ was found to have a more pronounced effect on the transient envelope than the constituent frequencies.

Figure 12 (top) shows a transient obtained at $\lambda_{\text {purn }}$ $=555 \mathrm{~nm}$ in a molecular beam expansion, showing a fundamental frequency very similar to that in Fig. 11(a), but generally more complex structure. The room temperature transients discussed above were fitted including only the nearest-neighbor term of Eq. (12). Such a fit, with freely varying $b$, is shown in Fig. 12 (middle). As expected, $b$ is an order of magnitude smaller than for the room temperature transients. However, the symmetric transient obtained from nearest neighbor coherences matches the experimental one only in its general features.

Figure 12 (bottom) shows a fit to the beam data including second-nearest neighbor coherences of frequency 

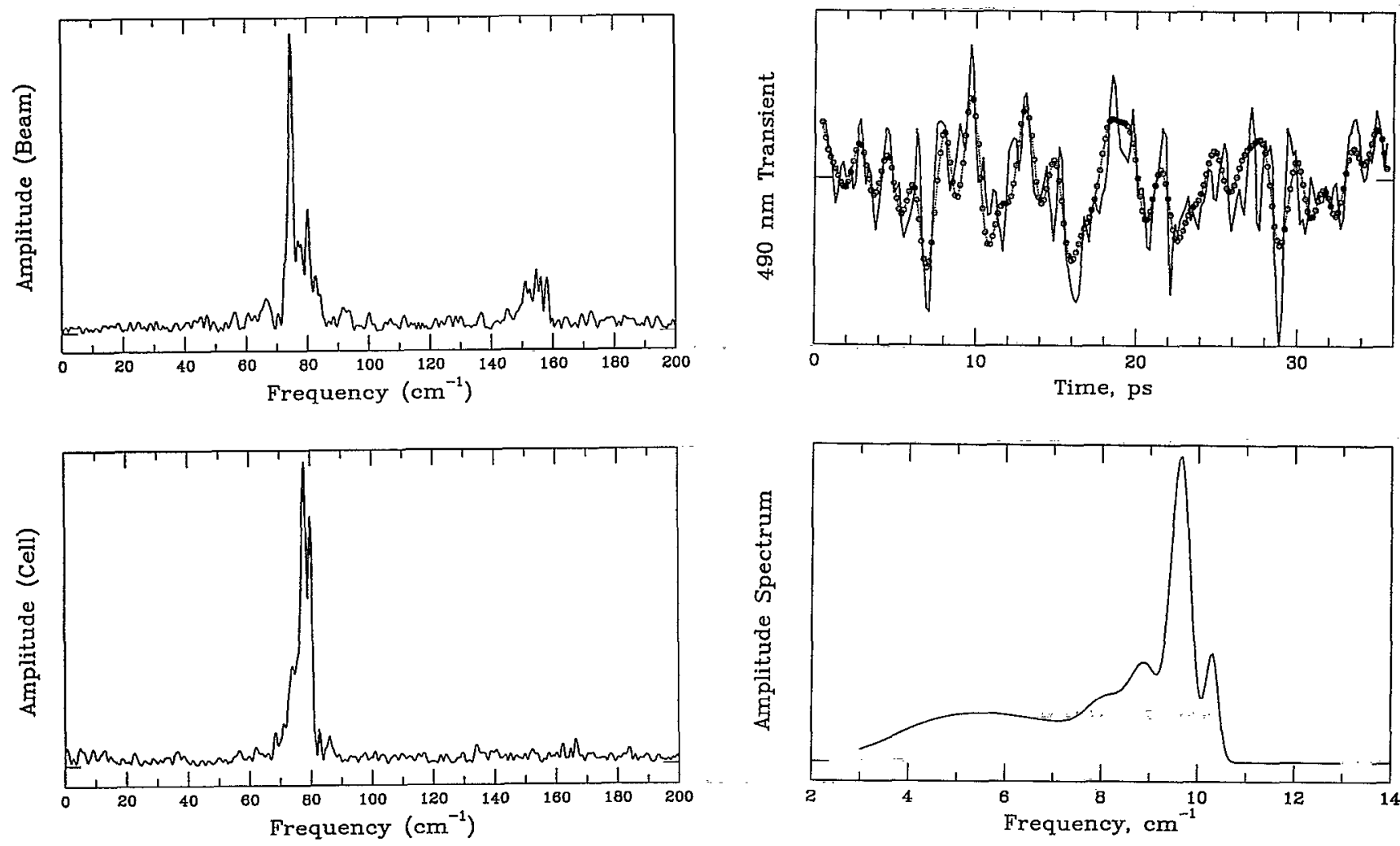

FIG. 13. Amplitude spectra of the beam transient at $555 \mathrm{~nm}$ and the room temperature transient at $550 \mathrm{~nm}$; the molecular beam transient shows a strong $2 \omega$ component which has not been averaged away by vibrational and rotational excitation of the initial density matrix (see the text).

$\approx 2 \omega$. It reproduces very well the asymmetry of the observed transients, and some of the $2 \omega$ beats, and yields similar frequencies. This is, however, at the expense of a much larger set of nonlinear fitting parameters (all the amplitudes and phases for the $2 \omega$ components). In effect, raising the temperature symmetrizes and simplifies the structure of the transient due to rotational and vibrational averaging, while preserving the desired information about the $1 \omega$ components with the same accuracy. Here, as opposed to the rotational coherences, it is a smoothing of the transient at higher temperatures that leads to a faster and simpler extraction of vibrational frequencies. Figure 13 shows Fourier amplitude spectra of the $\sim 550 \mathrm{~nm}$ transients in Figs. 11 and 12. The beam transient shows a much latger $2 \omega$ component. From the above discussion, it is clear that its size is not solely due to the laser response time, but also due to incoherent vibrational and rotational averaging at higher temperatures, which actually results in simpler coherence decays.

Finally, Fig. 14 shows the results that can be extracted from a very noisy transient, as a worst-case scenario for the nonlinear fitting technique. The vibrational frequencies extracted with the smallest uncertainty are 10.3(7) and $9.6(7) \mathrm{cm}^{-1}$, and could be assigned by extrapolating from the next smallest set near $15 \mathrm{~cm}^{-1}$ (see below). A number of other vibrational frequencies down to $4.3 \mathrm{~cm}^{-1}$ were

FIG. 14. Fit to a very noisy transient pumped at $\lambda=490 \mathrm{~nm}$, which lies mostly above the dissociation threshold; a sharp initial peak corresponding to dissociating $\mathrm{I}_{2}$ molecules is not shown and fitted; the fit reproduces the general structure of the transient, but as components higher than $2 \omega$ were not included, it cannot reproduce the sharper features in the transient.

also determined in the fit, but could not be assigned reliably due to the large uncertainty in the frequencies. This shows that meaningful results can still be extracted from noisy transients, with a concomitant loss in the accuracy of the information.

\section{INVERSION TO THE POTENTIAL}

In this section, we consider inversion of the data obtained above to the potential function (PF). Since this is closely related to the process of vibrational assignments, we also discuss this issue here.

\section{A. Vibrational assignments}

In cw spectroscopy, one is faced with the task of assigning specific lines to a complete set of quantum numbers. The wave packet approach does not bypass this problem. While the above analysis directly yields a spectrum of rotational constants $B_{v}$ and rotationless band origins $v_{v}$, a vibrational quantum number (and in general, several other quantum numbers) must still be assigned to the dual spectra. In the ideal case, assigning the rotational and vibrational constants to specific $v$ values is an easy task. Consider Fig. 15, showing the pure vibrational progressions obtained from the analysis is Sec. IV. As one approaches 


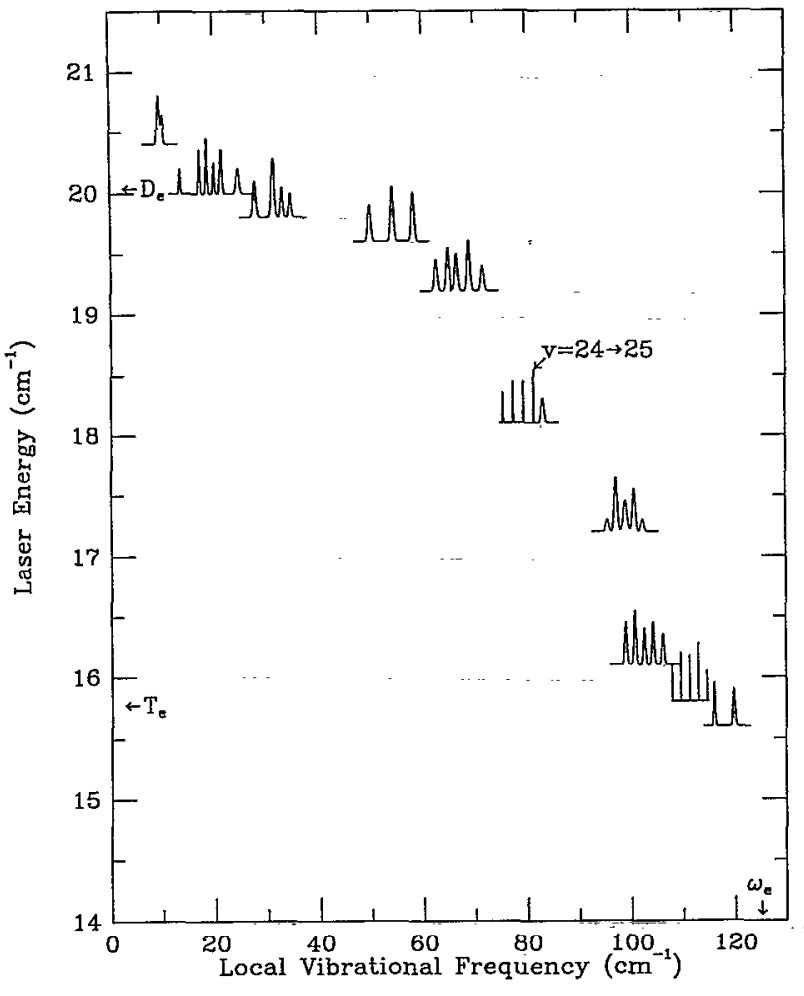

FIG. 15. Vibrational progression in the $B$ state of $I_{2}$ as measured by FWS: the curves are the amplitude spectra obtained from nonlinear fits to the experimental transients; the widths correspond to the estimated uncertainties; due to Franck-Condon factors and thermal excitation, the term energy and dissociation limit indicated by arrows do not perfectly line up with the laser energy.

the dissociation limit, the vibrational peaks crowd together as expected from higher order anharmonicity effects.

If the Franck-Condon factors are favorable, this progression can be followed to lower wavelengths, until the "first" vibrational peak is found, which can be assigned to $v=0$. Vibrations are then successively numbered up the ladder; a few missing rungs are not important, since only one assignment will generally bridge the gap without yielding grossly fluctuating local anharmonicity constants. In practice, the relative numbering is seldom a problem, but finding the $v=0$ peak can be. For instance, in $\mathbf{I}_{2}$ at room temperature, the transitions terminating below $v=4$ in the $B$ state are too weak to be detectable. Fortunately, vibrational transitions up to near dissociation are accessible, which constrains the vibrational level positions sufficiently to allow only one unique fit to a power series in $v+1 / 2$ without unduly large or fluctuating higher order terms in the Dunham expansion of the vibrational and rotational spectra.

The rotational assignment is usually possible in the same manner by working up a rotational ladder. In cases where the $B_{0}$ peak is not accessible, comparing peak intensities with the vibrational transient allows a unique assignment by a somewhat more detailed consideration of the factors $A_{V i j}$ and $A_{R i i}$ mentioned in Sec. III. As can be seen

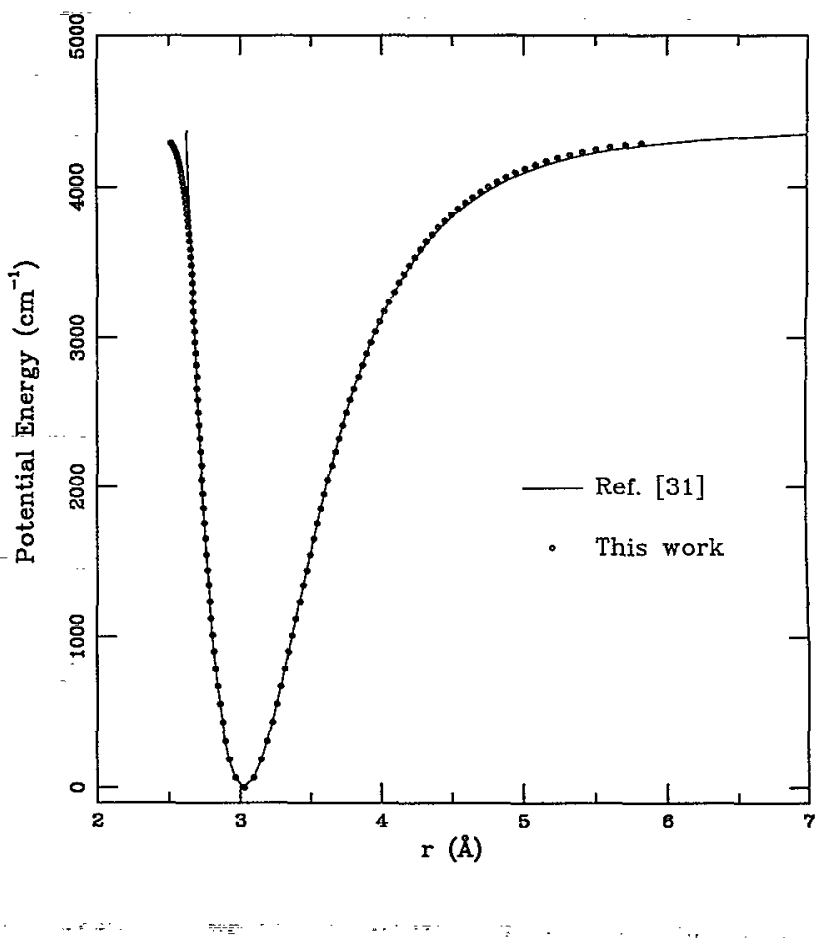

FIG. 16. High resolution and temporally derived RKR curves for the $B$ state of $\mathrm{I}_{2}$.

from Eqs. (2) and (8), the $A_{V i j}$ can be written as a product $c_{i} c_{j}$, while the $A_{R i i}$ can be written as products $c_{i}^{\prime 2}$; if the $J \pm 1$ splitting is not too large compared to the laser bandwidth, $c_{i}^{\prime} \approx c_{i}$, strongly correlating vibrational and rotational amplitudes.

Note that in principle one could also use a fit to the $c_{i}$ to determine the Franck-Condon factors between the two surfaces directly. The nearest neighbor and higher order amplitudes $A_{V i, i+1}, A_{V i, i+2} \cdots$ overdetermine the $c_{i j}$ yet in practice, the higher coherences are strongly damped by the laser bandwidth, which must be known accurately, and most importantly, the effects of rotation-vibration interaction; as a result, the $c_{i}$ cannot be fitted accurately except when the signal to noise ratio is very high and the excitation profile is well characterized.

\section{B. Potential functions}

A number of standard inversion procedures can be used once the vibrational frequencies and rotational constants have been determined. Table II summarizes the results of the measurements (including some older measurements from our previous work ${ }^{5}$ ), and the Dunham coefficients fitted to reproduce those measurements over the entire range of vibrational quantum numbers covered. We herc bricfly consider the RKR inversion ${ }^{18}$ and fitting to parameterized $\mathrm{PFs}^{19}$ as two methods of extracting the potential function from temporally obtained data.

Figure 16 summarizes the results for the $I_{2}$ PF obtained by RKR inversion; the error in the absolute energies 
TABLE II. Fitted Dunham expansion parameters; Uncertainties in parentheses are two standard deviations.

\begin{tabular}{|c|c|c|c|c|}
\hline \multirow{2}{*}{\multicolumn{2}{|c|}{ Dunham coefficients in $\mathrm{cm}^{-1}$}} & \multicolumn{3}{|c|}{ Observed and calculated rotational constants (in $\mathrm{cm}^{-1}$ ) } \\
\hline & & \multirow{2}{*}{$\frac{v}{6}$} & \multirow{2}{*}{$\frac{B(v)(\text { e. u. })}{0.02797(0.00002)}$} & \multirow{2}{*}{$\frac{B(\text { calc. })^{\mathrm{a}}}{0.0279697}$} \\
\hline$Y_{00}=$ & 0.15329 (calc.) & & & \\
\hline$Y_{10}=$ & $126.07(25)$ & 7 & $0.02780(0.00002)$ & 0.0277993 \\
\hline$Y_{20}=$ & $-0.825(35)$ & 8 & $0.02762(0.00002)$ & 0.0276253 \\
\hline$Y_{30}=$ & $0.002584(2271)$ & 9 & $0.02745(0.00002)$ & 0.0274475 \\
\hline$Y_{40}=$ & $-0.0002255(692)$ & 10 & $0.02727(0.00002)$ & 0.0272661 \\
\hline$Y_{\text {so }}=$ & $0.000003559(959)$ & 11 & $0.02708(0.00002)$ & 0.0270811 \\
\hline$Y_{60}=$ & $-0.00000001595(490)$ & 22 & $0.02481(0.00002)$ & 0.0248111 \\
\hline$Y_{01}=$ & $0.028986(107)$ & 23 & $0.02458(0.00002)$ & 0.0245839 \\
\hline$Y_{11}=$ & $-0.0001439(222)$ & 24 & $0.02434(0.00002)$ & 0.0243533 \\
\hline$Y_{21}=$ & $-0.0000019189(13787)$ & 25 & $0.02414(0.00002)$ & 0.0241193 \\
\hline \multirow[t]{2}{*}{$Y_{31}=$} & $0.000000003176(26718)$ & 26 & $0.02389(0.00002)$ & 0.0238820 \\
\hline & & 27 & $0.02363(0.00002)$ & 0.0236414 \\
\hline
\end{tabular}

Observed and calculated vibrational frequencies (in $\mathrm{cm}^{-1}$ )

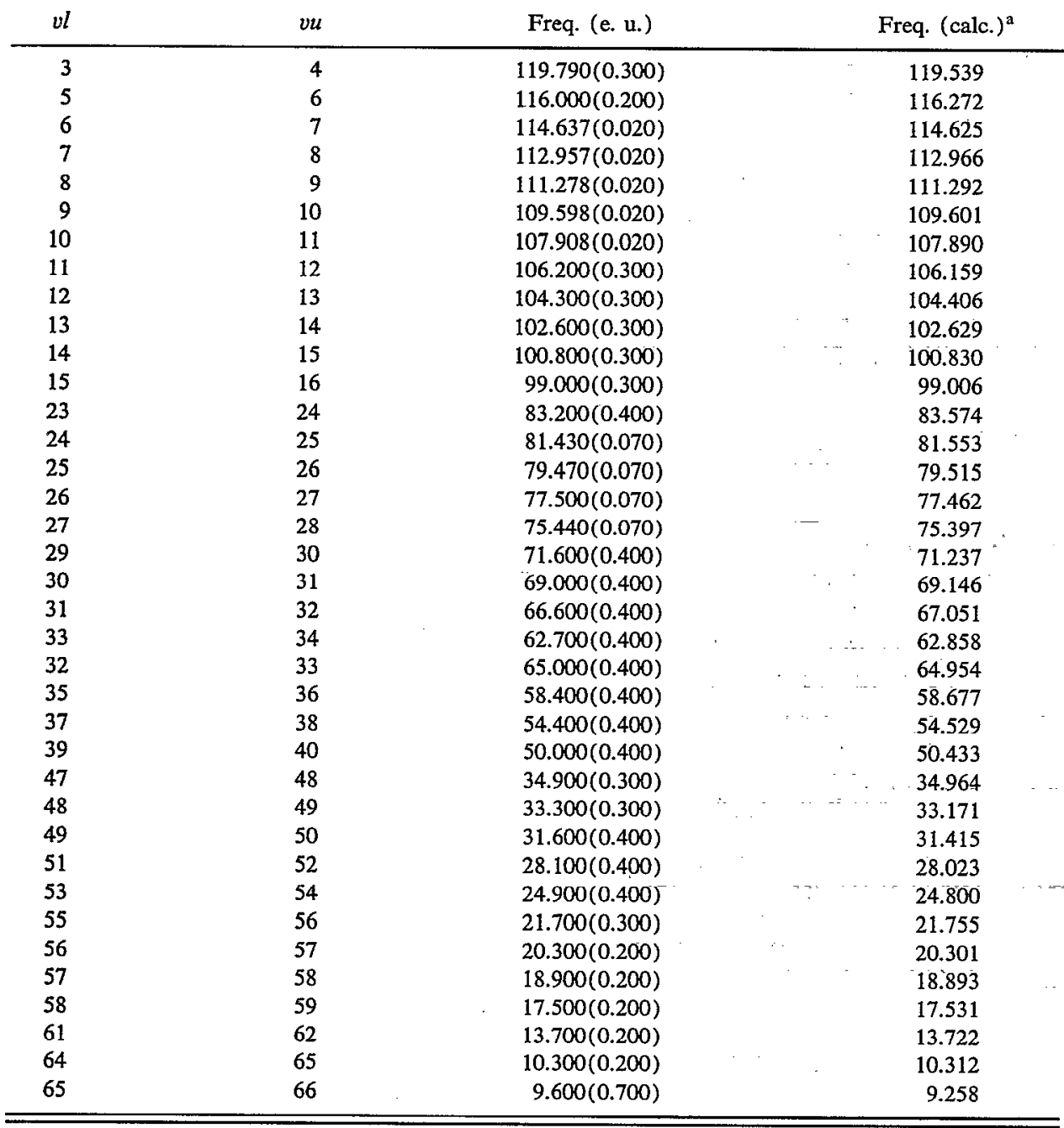

${ }^{2} e$. $u$. is the estimated 2 sigma uncertainty; $v l$ and $v u$ are the lower and upper vibrational quantum numbers fitted to a specific frequency component of a transient.

(measured from the bottom of the $B$-state well), amounts to $3 \mathrm{~cm}^{-1}$ at $v=65,65 \mathrm{~cm}^{-1}$ below the dissociation limit. The error in the turning point difference, $r_{\text {outer }}-r_{\text {inner }}$, is similarly small up to $v=70$. The actual turning points tend to shift increasingly to smaller internuclear distances above $v=50$, due to the lack of rotational sampling above $v=30$. A number of methods can circumvent this artifact of the RKR inversion. One that also yields a continuously defined potential involves fitting the rotational constants and vibrational frequencies directly to a model potential; a 
TABLE III. $f$ potential parameters for the $B$ state of $I_{2}$; Values of $p$ 's and $q$ 's are such that $r$ is in Angstroms (see the text).

\begin{tabular}{ll}
\hline \hline$D$ & $4381.5 \mathrm{~cm}^{-1}$ \\
\hline$p_{0}$ & 1.0 \\
$p_{2}$ & -2.0 \\
$p_{4}$ & 0.68529208 \\
$q_{2}$ & 7.9 \\
$q_{4}$ & -8.2429158 \\
$q_{6}$ & 1.9815692 \\
$q_{8}$ & 0.24525151 \\
$q_{10}$ & 0.061353379 \\
$q_{12}$ & -0.108471 \\
\hline
\end{tabular}

particularly convenient function is the " $f$ potential" of Ref. 19,

$$
V(r)=D[1-f(r)]^{2}
$$

which fits the data to a smoothly and monotonically decreasing mapping function; the following Padé approximant for $f$ was found sufficient in this case,

$f(r)$

$$
=\frac{p_{0}+p_{2} r+p_{4} r^{2}}{q_{1} r^{1 / 2}+q_{2} r+q_{4} r^{2}+q_{6} r^{3}+q_{8} r^{4}+q_{10} r^{5}+q_{12} r^{6}+q_{14} r^{7}},
$$

where $r$ is the internuclear distance. If we constrain two of the coefficients to be

$$
q_{1}=2.9343 \times 10^{-3}(D / Z)^{2} p_{0} \text { and } q_{14}=2 D / C_{5} p_{4} \text {, }
$$

where $D$ is the dissociation energy in $\mathrm{cm}^{-1}, Z$ the nuclear charge (127), and $C_{5}$ the van der Waals coefficient (3.11 $\times 10^{5} \mathrm{~cm}^{-1} \AA^{5}$ ) for the $B$ state of $I_{2}$, then the PF will have the correct $1 / r^{5}$ behavior at large internuclear distances, ${ }^{20}$ and the correct (shielded) Coulombic behavior at small $r$. The resulting fitted constants $q_{i}$ and $p_{i}$ are shown in Table III, and the potentials are compared in Fig. 17. Much better behavior is found for the $f$-potential near the dissociation limit, yielding a continuous global potential function with only a few adjustable parameters.

\section{COMPARISON WITH CLASSICAL AND QUANTUM DYNAMICS}

The classical treatment of the temporal data and their inversion to the potential have been discussed by Bernstein and Zewail. ${ }^{6}$ Basically, the local period can be related to the difference in the turning points of the potential. At all wavelengths, experiments and classical calculations agree. However, the classical treatment does not give as precise information as the quantum calculation because of structure in the wave packet.

In our previous letter, we reported a time-dependent wave packet calculation on the RKR potential obtained therein..$^{5}$ Engel and Metiu reported calculation on model surfaces of the $E$ state in addition to the $f$ state. ${ }^{21}$ In our calculations; it was shown that the RKR potential yielded simulated vibrational transients which in turn had vibra-
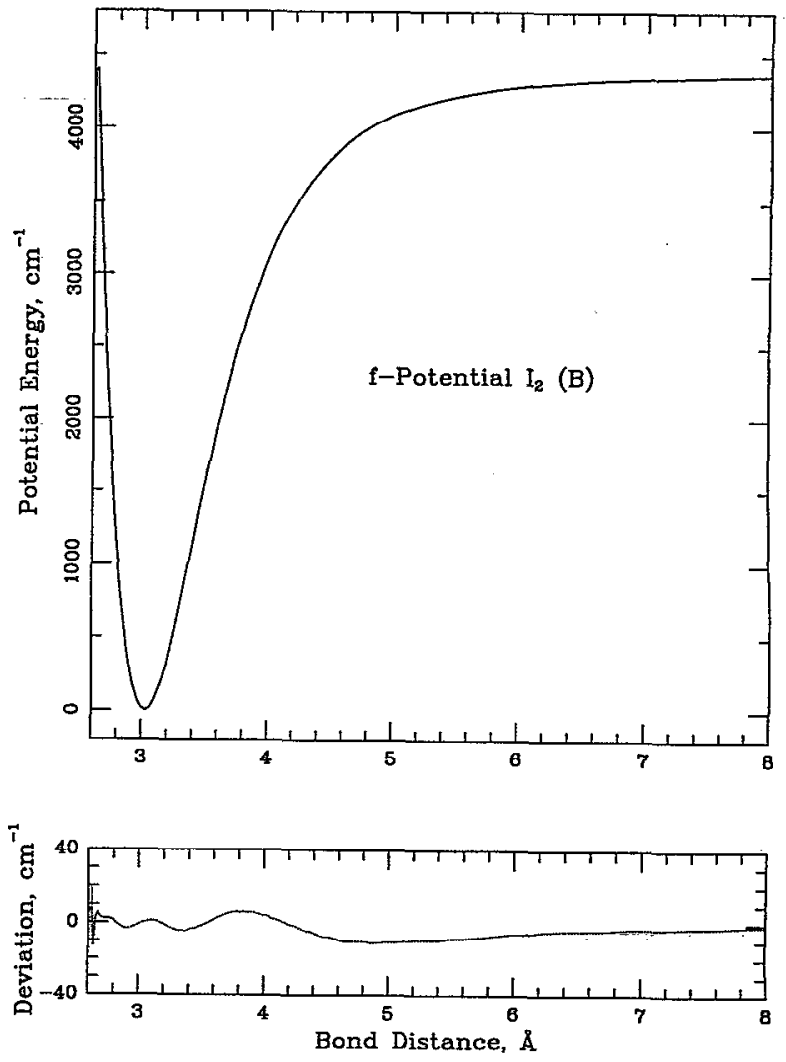

FIG. 17. $f$ potential for $\mathrm{I}_{2}$ derived from the present data, and difference potential to the RKR potential from Ref. 31 .

tional frequency components very close to those determined in the experiment.

Here our goal is not to demonstrate self-consistency again, which can be assumed to hold better now than in the earlier potential determination, since the actual observed transients can be simulated within experimental unccrtainty. Rather, we perform a series of pump-probe calculations on simple, approximate Morse-model potentials for the $X, B$, and $f$ states, to investigate the general effects of vibrational and rotational excitation in the $X$ state on the transients. The goal is to see whether the trends ascribed to the various room temperature and molecular beam experiments for the coherence transients are indeed borne out by theoretical simulations.

We consider only total fiuorescence from the $f$ state. This greatly simplifies the calculations, since only the amplitude of the final wave packet need be considered, rather than a weighted sum of the type shown in Eq. (5). In the experiments described in Ref. 8, the $E$ state should not play a great role, since fluorescence in the experiments was detected only near $340 \mathrm{~nm}$, which can result only from the $f$ state directly, or via collisional quenching from it to the $D^{\prime}$ state.

The time-dependent quantum formalism, discussed by Kosloff and Hammerich, ${ }^{22}$ Williams and Imre, ${ }^{23}$ Engel and Metiu, ${ }^{24}$ Hartke and Manz, ${ }^{25}$ and others ${ }^{22}$ has been applied by us previously to $I_{2}$ (Ref. 5) and several other molecules, ${ }^{26}$ and need not be rederived here in detail. The 


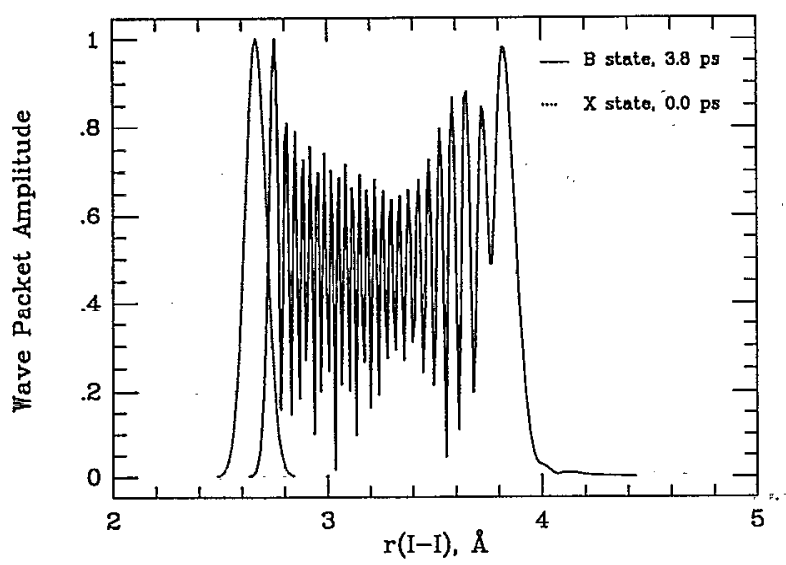

FIG. 18. Initial $v=0$ wave packet, and wave packet 3.8 ps after a $552 \mathrm{~nm}$ pump pulse in the $B$ state of $I_{2}$. It has spread through the entire energetically accessible part of the potential well, and the node number indicates it is composed of vibrational eigenstates near $v=25$.

present experiments can be treated by second order perturbation theory of the pump-probe matter-radiation Hamiltonian; we chose laser pulse widths of $90 \mathrm{fs}$ (FWHM Gaussian, taken in time to $\pm 2 \sigma$ ), to match a temporal width. Wave packets were propagated using the Feit and Fleck split-operator technique. ${ }^{27}$

Figure 18 shows the original ground state wave function $(v=0)$ and the $B$ state wave packet 3.8 ps after termination of the pump pulse at $552 \mathrm{~nm}$. As one might expect, the wave packet displays signs of nodal structure near $v=25$, which is the approximate vibrational quantum number of the eigenstates with the largest contribution to the wave packet. Figure 19 shows a longer time autocorrelation of the same wave packet, as well as its potential and kinetic energy expectation values. It can be seen that after about $10 \mathrm{ps,}$, the packet behaves least like a single classical particle, being sufficiently delocalized to damp out fluctuations in the kinetic and potential energies.

Due to the large masses of the iodine atoms, the coherence behavior could be expected to be mimicked fairly closely by an ensemble of classically propagated particles with appropriate initial conditions. Figure 20 (top) shows the autocorrelation of the classical probability density functional $f[(x(t), p(t)]$ for 1000 particles. The initial position distribution used corresponds to the upper state wave packet density after the pump pulse from $v=0$ is over, and the continuous energy distribution is given by the laser profile. (We have chosen this initial distribution rather than a Wigner function because it is positive definite, and still fairly accurately reproduces the local energy vs position dependence of the initial wave packet in the $B$ state.) This dephases after a few vibrational periods due to the lack of quantum coherence between discrete vibrational energy levels, and does not closely resemble the quantum autocorrelation function or the experimental transients. Figure 20 (bottom) shows a similar calculation, with the particles constrained to the discrete eigenvalues of our Morse model potential for the $B$ state. This autocorrelation qualitatively resembles the quantum autocorrelation and
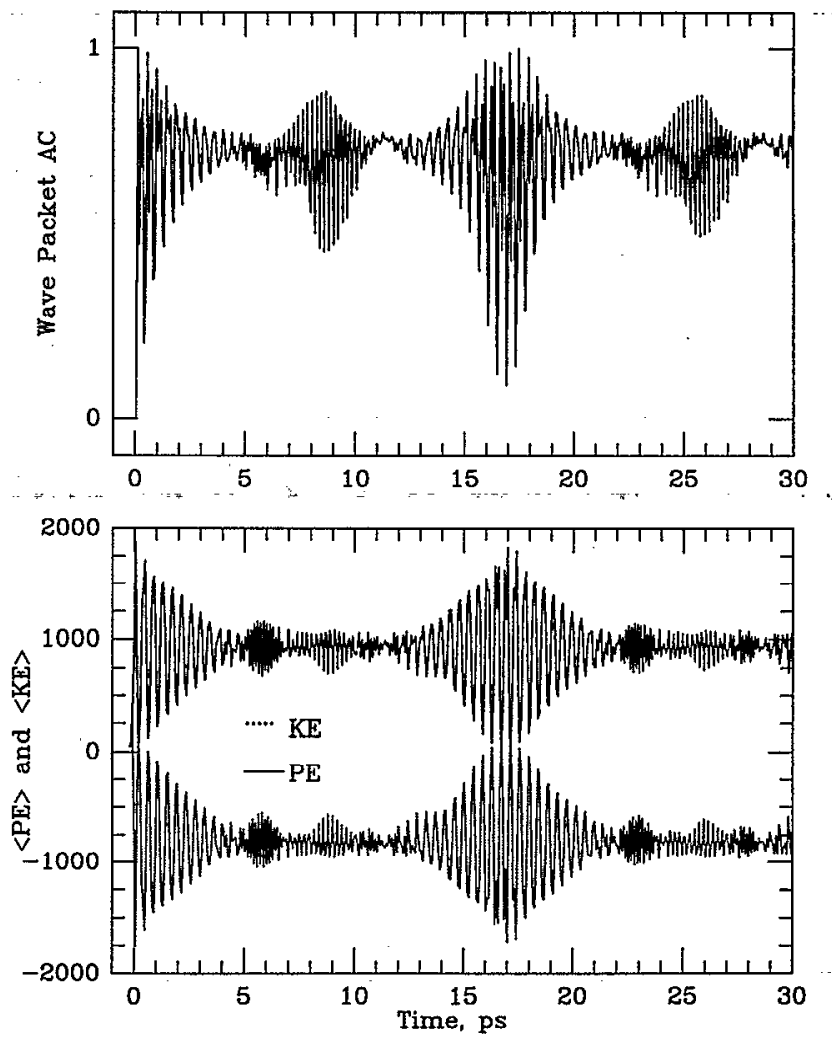

FIG. 19. Wave packet calculations on $I_{2}$; the upper figure shows an autocorrelation of the $B$ state wave packet; the more "random" zones at 5,11 , and 22 ps correspond to a wave packet that has spread over the entire well and is quantal in nature; the lower figure shows the corresponding kinetic and potential energy expectation values.

experimental results, and shows rephasing due to the anharmonicity of the vibrational energy levels. Thus, at the very least a simple semiclassical model is required to model the experimentally observed transients.

We now return to the quantum calculations, and consider the effects of rotational and vibrational excitation on the calculated fluorescence signal from the $f$ state. Figure 21 shows the effects on the vibrational transient of vibrational excitation in the absence of rotational excitation; the total fluorescence spectra pumped at $552 \mathrm{~nm}$ and probed at $310 \mathrm{~nm}$ are shown for initial vibrational levels of 0,1 , and 2. The fluorescence becomes increasingly structured with more nodes present in the initial wave function. The bottom of Fig. 21 shows a thermally $(300 \mathrm{~K})$ weighted average, which is considerably smoothed out. As expected, the second anharmonicity maximum near $17 \mathrm{ps}$ (for the $B$ state model surface) has the same amplitude as the $\tau=0$ maximum, since there is no rotational damping. At lower temperatures, the transient becomes increasingly like the $v=0$ one, showing more structure in the "dephased" region between maxima.

Figure 22 similarly shows the effects of rotational excitation on the transients. At the bottom is shown the result of a thermal average (with transients at $J$ values other than $0,30,60,90$ interpolated along both the amplitude and 

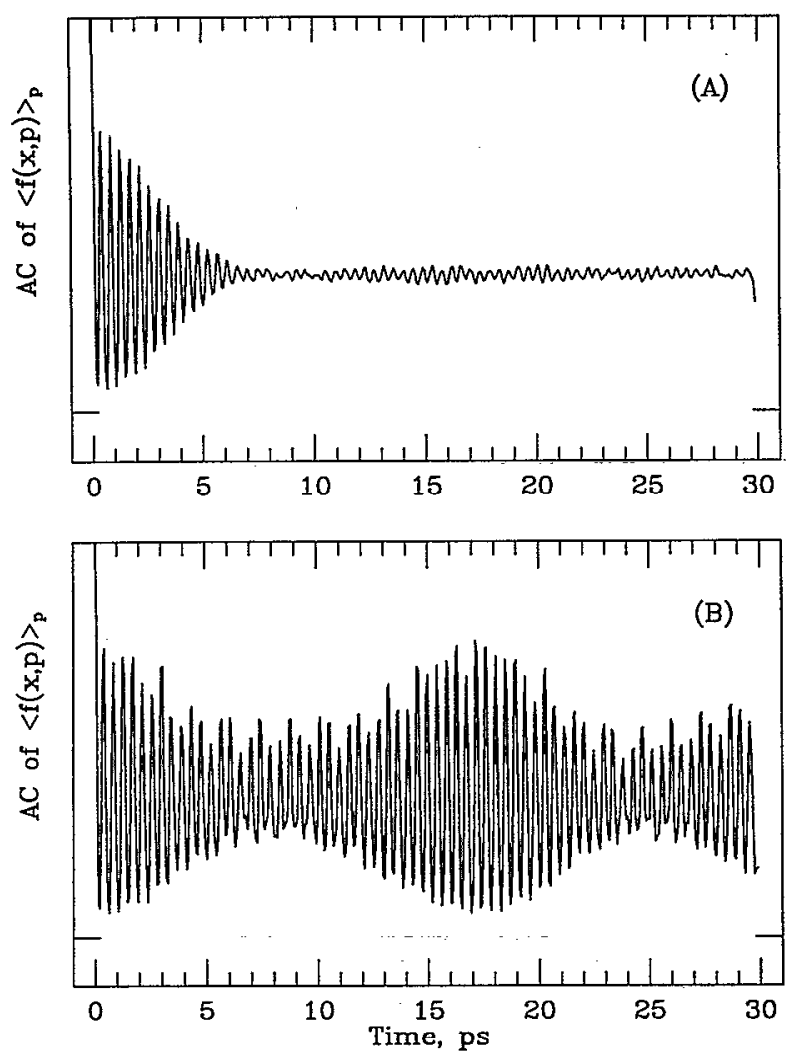

FIG. 20. Classical density functional autocorrelation functions for different initial conditions; the upper figure shows the autocorrelation for a set of particles spread over a continuous energy range within the laser bandwidth; the lack of coherence without a set of discrete energy levels is evident in the complete dephasing of the trace after several vibrational periods; the lower trace shows the autocorrelation for particles constrained to a discrete set of initial energies corresponding to the vibrational levels of the $B$ state; as one might expect for a molecule as massive as $I_{2}$, this simple semiclassical approximation at least qualitatively reproduces the features of the quantum autocorrelation and experimental fluorescence transients.

time axes), with the damping effect, due to the slight continuous variation of the local vibrational frequency with $J$, clearly visible. The higher $J$ transients also show a somewhat simpler behavior, with a "washed-out" region where the wave packet has dephased, as observed in the experimental room temperature transients.

Thus, the quantum simulations qualitatively bear out the smoothing of vibrational transients observed at higher temperatures, the effect of which is to allow simpler and more accurate fitting of the transients, rather than causing loss of information. While vibrational averaging alone can account for some smoothing, rotational averaging is required to explain the rapid damping of the "beat" maxima in the transient.

As a final point, we consider the effects of laser pulse shape and incoherence (nontransform limit) on the transients. We performed these calculations in the frequency domain, using the known FC factors (for the $X-B$ transition) and energy levels. Figures 23(a) and 23(b) show a full simulation of the vibrational and rotational recurrences obtained with jitter-free, transform-limited Gauss-
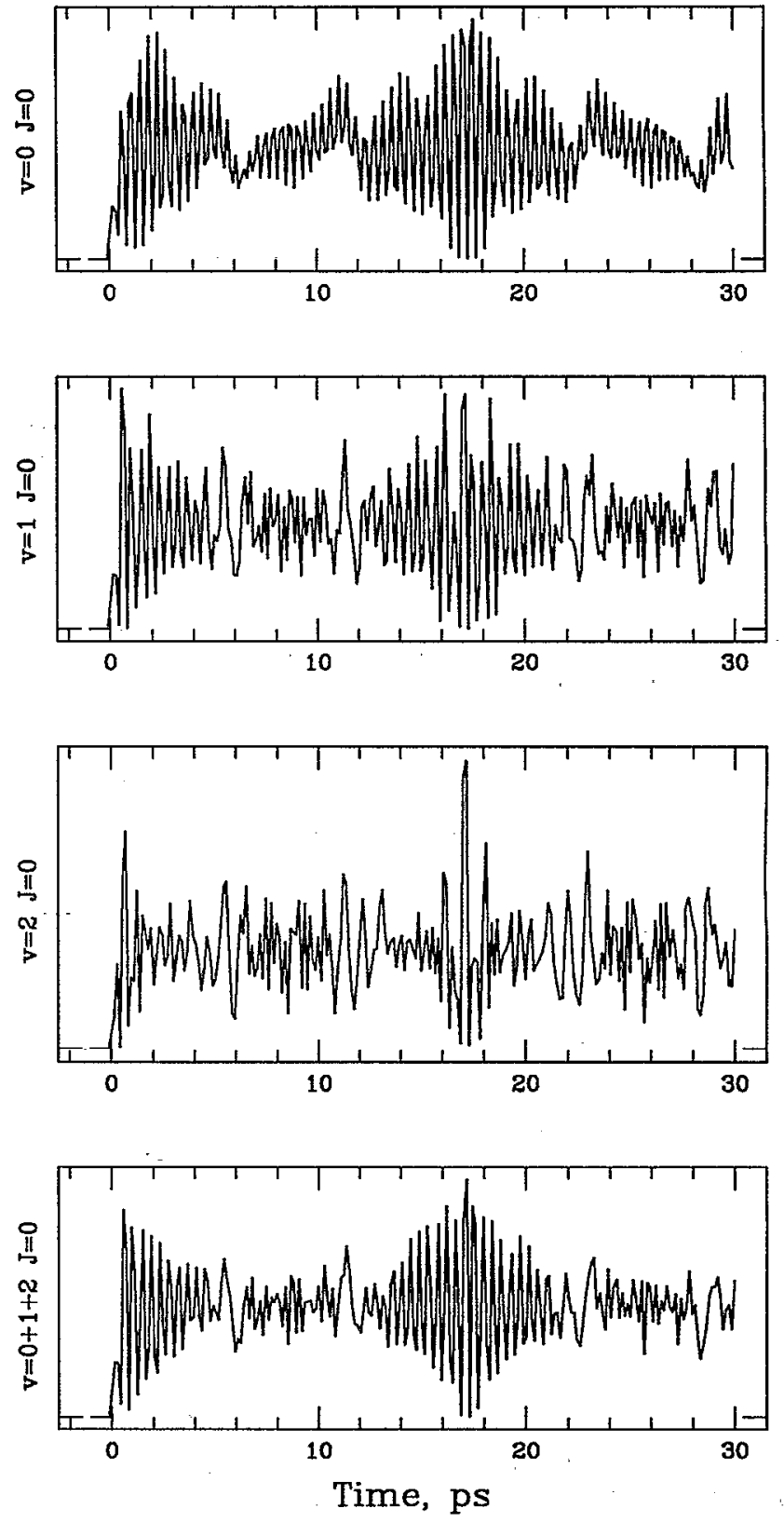

FIG. 21. Vibrational dependence of the detected fluorescence transient; the complexity of the structure is related to the complexity of the initial wave function; thermal averaging removes much of this structure (some slight irregularities are due to aliasing since the fluorescence spectrum was calculated at intervals of $67 \mathrm{fs}$ ).

ian pulses of FWHM 90 fs, using Eqs. (8) and (9). The main features of the magic angle transient resemble those of both the quantum-dynamics simulation and the $550 \mathrm{~nm}$ experiments, the differences being due to different assumptions about FC factors, the Morse approximation, or the different nature of the experimental laser pulses. The "noise" in the rotational transient $23 b$ is remarkable in that it is also found experimentally, and is due to a highly dephased vibrational wave packet.

Figure 24(a) shows a similar calculation for non transform-limited pulses with a 90 fs FWHM Gaussian 

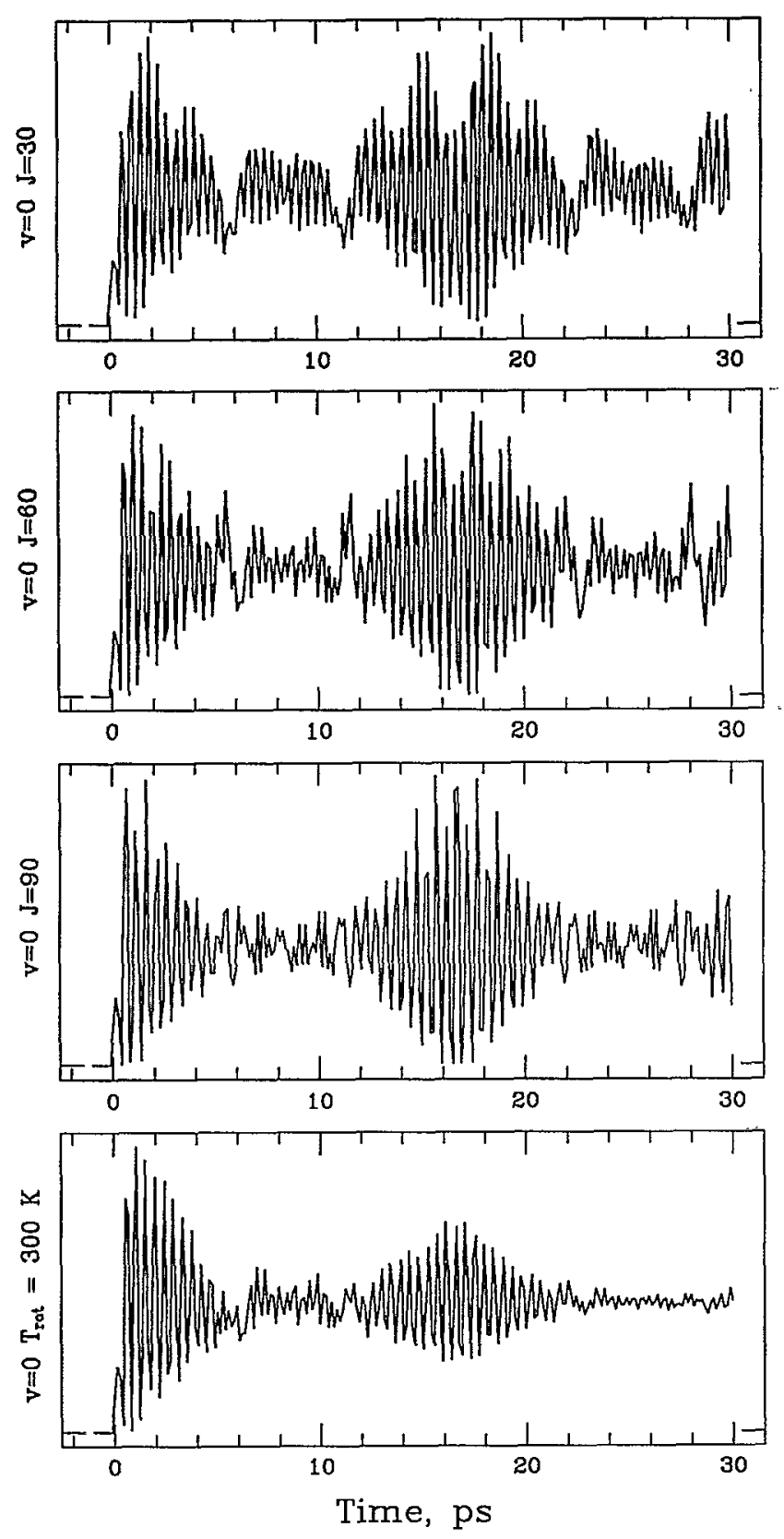

FIG. 22. Rotational dependence of the detected fluorescence transient; the rotational averaging is seen to lead to the damping described analytically by Eq. (14).

temporal profile and a $10 \mathrm{~nm}$ square bandwidth profile (typical of continuum generation). Fig. 24b shows a transform-limited pulse with a $10 \mathrm{~nm}$ square bandwidth profile for comparison. These differences show how sensitive the transients are to the exact amplitudes $A_{V i j}$ [and the partially randomized phase $\Delta \phi$ in Eq. (8)], even when the constituent frequencies are nearly identical. For example, the larger temporal width of the pulses in Fig. 24(a), compared to Fig. 24(b), leads to a clear reduction in higher frequency beats arising from more-than-once removed neighboring vibrational levels. The effects of pulse shape, phase, and multiplicity on coherent transients have been
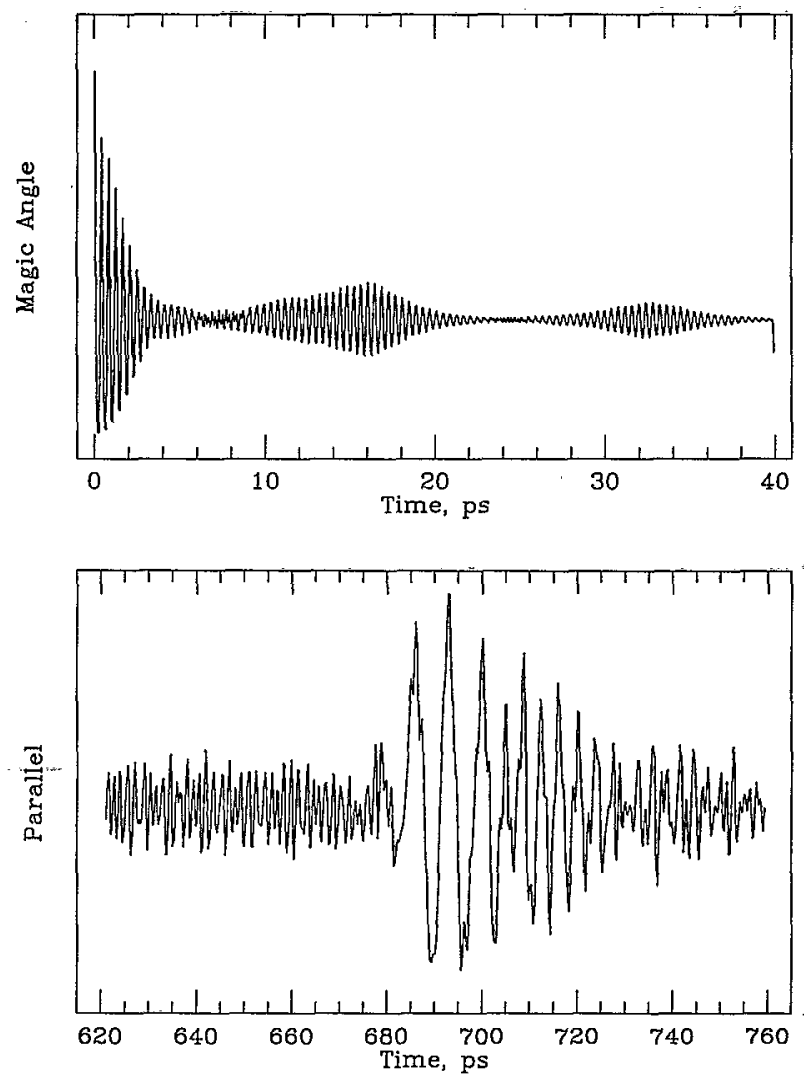

FIG. 23. Vibrational (magic angle) and rotational (parallel polarizations, full recurrence) transients calculated using Eqs. (8) and (9) for a Gaussian transform-limited beam of FWHM 90 fs.

extensively studied in nanosecond, ${ }^{28}$ picosecond, ${ }^{29}$ femtosecond experiments, ${ }^{30}$ and in theory. ${ }^{32}$

\section{CONCLUSIONS}

Femtosecond wave packet dynamics in isolated (collisionless) systems are discussed in relation to coherences (vibrational and rotational), vibration-rotation spectra and inversion to the potentíal. Experimental and theoretical results are presented for iodine, as an example, in gasphase cells and in molecular beams. Other relevant systems are given in the work by the Gerber's group ${ }^{33}$ and in Ref. 1. A number of interesting effects contribute to the appearance of FWS transients, and were considered here in attempts to quantify the dependence of the observed dynamics on coherences of nuclear vibrational and rotational motions.

Because FWS critically depends on coherent preparation of wave packets on a given potential, the method offers some unique features: (a) the separation of time scales for vibrational and rotational spectra (from measurements of scalar and vector properties of the dynamics), simplifying the analysis considerably; (b) the examination of only the potential on which coherence is formed (not the other two involved in the transitions), minimizing inhomogeneous broadening effects and providing information on spectrally congested, thermally heated samples; and (c) the ability to directly study the potential and spectra of bound intermediates in a chemical reaction. Some of the approaches pre- 

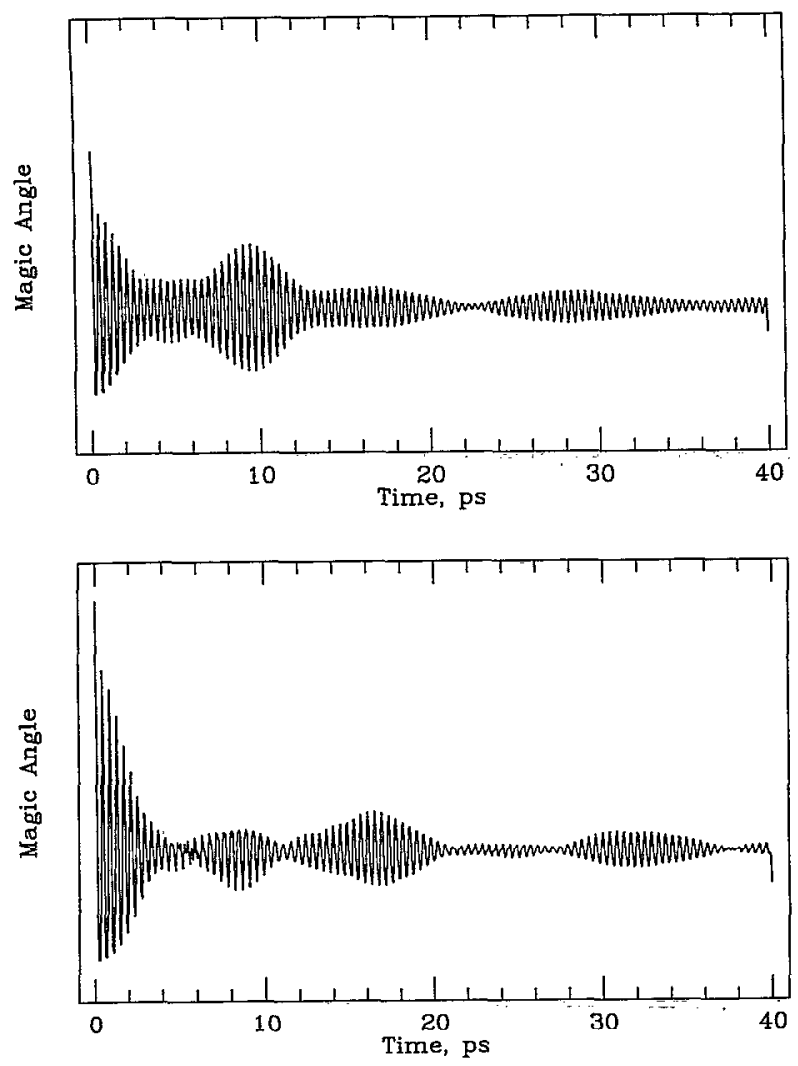

FIG. 24. Vibrational transients analogous to Fig. 23, but for laser pulses with gaussian temporal width of $90 \mathrm{fs}$ and $10 \mathrm{~nm}$ square wavelength profile (upper) and same wavelength profile, but transform limited (lower).

sented here should prove useful in applications to complex systems where multiple potentials are involved or the dimensionality of the PES cannot be reduced to one.

\section{ACKNOWLEDGMENT}

This work was supported by a grant from the U.S. Air Force Office of Scientific Research. We thank Soren Pedersen for careful reading of the manuscript, and Jennifer Herck for her help.

${ }^{1}$ See: A. H. Zewail, Science 242, 1645 (1988); L. R. Khundkar and A. H. Zewail, Ann. Rev. Phys. Chem. 41, 15 (1990); A. H. Zewail, Faraday Discuss. Chem. Soc. 91, 207 (1991), and references therein.

${ }^{2}$ See: P. M. Felker and A. H. Zewail, Adv. Chem. Phys. 70, 265 (1988); M. Cote, J. F. Kaufmann, P. G. Smith, and J. D. McDonald, J. Chem. Phys. 90, 2865 (1989); W. R. Lambert, P. M. Felker and A. H. Zewail, ibid. 75, 5958 (1981); A. J. Kaziska and M. R. Topp, Chem. Phys. Lett. 180, 423 (1991); M. Ivanco, J. Hager, W. Sharfin, and S. C. Wallace, J. Chem. Phys. 78, 6531 (1983); J. M. Smith, C. Lakshminarayan, and J. L. Knee, ibid. 93, 4475 (1990); P. M. Felker, J. Phys. Chem. 96, 7844 (1992), and references therein.

${ }^{3}$ M. Dantus, R. M. Bowman, and A. H. Zewail, Nature (London) 343, 737 (1990).

${ }^{4} \mathrm{H}$. Bitto and J. R. Huber, Acc. Chem. Res. 25, 65 (1992), and references therein.

${ }^{5}$ M. Gruebele, G. Roberts, M. Dantus, R. M. Bowman, and A. H. Zewail, Chem. Phys. Lett. 166, 459 (1990).
${ }^{6}$ M. II. M. Janssen, R. M. Bowman, and A. H. Zewail, Chem. Phys. Lett. 172, 99 (1990); R. B. Bernstein and A. H. Zewail, ibid. 170, 321 (1990).

${ }^{7}$ S. Gerstenkorn and P. Luc, J. Phys. (Paris) 46, 867 (1985); R. S. Mulliken, J. Chem. Phys. 55, 288 (1971).

${ }^{8}$ R. M. Bowman, M. Dantus and A. H. Zewail, Chem. Phys. Lett. 161, 297 (1989).

${ }^{9}$ See J. C. D. Brand and A. R. Hoy, Can. J. Phys. 60, 1209 (1982); R. J. Donovan, A. J. Holmes, P. R. R. Langridge-Smith, and T. Ridley, J. Chem. Soc. Faraday Trans. 85, 541 (1988).

${ }^{10}$ P. M. Felker and A. H. Zewail, J. Chem. Phys. 86, 2460 (1987); Adv. Chem. Phys. 70, 265 (1988).

${ }^{11}$ R. M. Bowman, M. Dantus, and A. H. Zewail, Chem. Phys. Lett. 174, 546 (1990).

${ }^{12}$ M. Dantus, M. H. M. Janssen, and A. H. Zewail, Chem. Phys. Lett. 181, 281 (1991).

${ }^{13}$ E. D. Potter, J. Herek, Q.-L. Liu, S. Pedersen, and A. H. Zewail, Nature (London) 355,"66 (1992).

${ }^{14}$ A. B. Myers and R. M. Hochstrasser, J. Chem. Phys. 85, 6301 (1986), and references therein.

${ }^{15}$ G. V. Hartland, L. L. Connell, and P. M. Felker, J. Chem. Phys. 94, 7649 (1991); G. V. Hartland, P. W. Joireman, L. L. Connell, and P. M. Felker, ibid. 96, 179 (1992).

${ }^{16}$ S. Mukamel, Ann. Rev. Phys. Chem. 41, 647 (1990); Density Matrix Methods and Femtosecond Processes, edited by S. H. Lin (World Scientific, Singapore, 1991); W. T. Pollard, H. L. Fragnito, J. Y. Bigot, C. V. Shank, and R. A. Mathies, Chem. Phys. Lett. 168, 239 (1990).

${ }^{17}$ S. M. Kay and S. L. Marple, Jr., Proc. IEEE 69, 1380 (1981).

${ }^{18}$ C. Schwartz and R. J. Leroy, J. Chem. Phys. 81, 3996 (1984), and references therein.

19 M. Gruebele, Mol. Phys. 69, 475 (1990).

${ }^{20}$ R. J. Le Roy and R. B. Bernstcin, J. Mol. Spectrose. 37, 109 (1971).

${ }^{21}$ H. Metiu and V. Engel, J. Chem. Phys. 93, 3252 (1990).

${ }^{22}$ R. Kosloff and A. Dell Hammerich, Faraday Discuss. Chem. Soc. 91, 239 (1991), and references therein.

${ }^{23}$ S. O. Williams and D. G. Imre, J. Chem. Phys. 92, 6636, 6648 (1988).

${ }^{24}$ V. Engel, H. Metiu, R. Almeida, R. A. Marcus, and A. H. Zewail, Chem. Phys. Lett. 152, 1 (1988); V. Engel and H. Metiu, J. Chem. Phys. 90, 6116 (1989); Ch. Meier, V. Engel and J. S. Briggs, ibid. 95, 7337 (1991).

${ }^{25}$ B. Hartke, J. Manz, and J. Mathis, Chem. Phys. 139, 123 (1989); B. Hartke, Chem. Phys. Lett. 175, 322 (1990).

${ }^{26}$ M. Gruebele, G. Roberts, and A, H. Zewail, Philos. Trans. R. Soc. London, Ser. A 332, 223 (1990); G. Roberts and A. H. Zewail, J. Phys. Chem. 95, 7973 (1991).

${ }^{27}$ M. D. Feit, J. A. Fleck, Jr., and A. Steiger, J. Comput. Phys. 47, 412 (1982).

${ }^{28}$ W. S. Warren and A. H. Zewail, J. Chem. Phys. 75, 5956 (1981); 78, 2279 (1983).

${ }^{29}$ J. P. Heritage, T. K. Gustavson, and C. H. Lin, Phys. Rev. Lett. 34, 1299 (1975); W. S. Warren, Science 242, 876 (1988).

${ }^{30}$ N. F. Scherer, A. J. Ruggiero, M. Du, and G. R. Fleming, J. Chem. Phys. 93, 856 (1990); N. F. Scherer, R. J. Carlson, A. Matro, M. Du, A. J. Ruggiero, V. Romerorochin, J, A. Cina, G. R. Fleming, and S. A. Rice, ibid. 95, 1487 (1991); A. M. Weiner, J. P. Heritage and E. M. Kirschner, J. Opt. Soc. Am. 85, 1563 (1988); A. M. Weiner, D. E. Leaird, G. P. Wiederrecht, and K. A. Nelson, Science 247, 1317 (1990); J. Gerdy, M. Dantus, R. Bowman, and A. H. Zewail, Chem. Phys. Lett. 171, 1 (1990).

${ }^{31}$ R. F. Barrow and K. K. Yee, J. Chem. Soc. Faraday Trans. 2 69, 684 (1973).

${ }^{32}$ For a review, see D. J. Tannor and S. A. Rice, Adv. Chem. Phys. LXX (Part 1), 441 (1988); Also, J. Cina, Phys. Rev. Lett. 66, 1146 (1991); E. Kolba and J. Manz, Faraday Discuss. Chem. Soc. 91, 369 (1991), and papers in this Discussion volume. For work on the theoretical consideration of multiple pulse and phase-coherent pulses, sec also Ref. 28.

${ }^{33}$ T. Banmert, V. Engel, C. Röttgermann, W. T. Strunz, and G. Gerber, Chem. Phys. Lett. 191, 639 (1992); J. Phys. Chem. 95, 8103 (1991); T. Banmert, M. Grosser, R. Thalweiser, and G. Gerber, Phys. Rev. Lett. 67, 3753 (1992). 